\title{
The properties of radio and mid-infrared detected galaxies and the effect of environment on the co-evolution of AGN and star formation at $z \sim 1$
}

\author{
Lu Shen ${ }^{\ominus}, 1,2,3 \star$ Brian C. Lemaux ${ }^{\odot},{ }^{1}$ Lori M. Lubin, ${ }^{1,4} \dagger$ John McKean, ${ }^{5}$ Neal \\ A. Miller, ${ }^{6}$ Debora Pelliccia, ${ }^{1,7}$ Christopher D. Fassnacht, ${ }^{1}$ Adam Tomczak ${ }^{\oplus},{ }^{1}$ \\ Po-Feng Wu, ${ }^{8}$ Dale Kocevski, ${ }^{9}$ Roy Gal, ${ }^{10}$ Denise Hung ${ }^{\oplus 10}$ and Gordon Squires ${ }^{11}$ \\ ${ }^{1}$ Physics Department, University of California, Davis, One Shields Avenue, CA 95616, USA \\ ${ }^{2}$ CAS Key Laboratory for Research in Galaxies and Cosmology, Department of Astronomy, University of Science and Technology of China, Hefei 230026, \\ China \\ ${ }^{3}$ School of Astronomy and Space Sciences, University of Science and Technology of China, Hefei 230026, China \\ ${ }^{4}$ The Observatories, The Carnegie Institution for Science, 813 Santa Barbara St, Pasadena, CA 91101, USA \\ ${ }^{5}$ Kapteyn Astronomical Institute, University of Groningen, NL-9700 AB Groningen, the Netherlands \\ ${ }^{6}$ Department of Mathematics and Physics, Stevenson University, 1525 Greenspring Valley Road, Stevenson, MD 21153, USA \\ ${ }^{7}$ Department of Physics and Astronomy, University of California, Riverside, 900 University Avenue, Riverside, CA 92521, USA \\ ${ }^{8}$ National Astronomical Observatory of Japan, Osawa 2-21-1, Mitaka, Tokyo 181-8588, Japan \\ ${ }^{9}$ Colby College, 4000 Mayflower Hill, Waterville, ME 04901, USA \\ ${ }^{10}$ Institute for Astronomy, University of Hawai'i, 2680 Woodlawn Drive, Honolulu, HI 96822, USA \\ ${ }^{11}$ Spitzer Science Centre, California Institute of Technology, M/S 220-6, 1200 E. California Blvd, Pasadena, CA 91125, USA
}

Accepted 2020 April 7. Received 2020 April 2; in original form 2019 October 7

\begin{abstract}
In this study, we investigate 179 radio-infrared (IR) galaxies drawn from a sample of spectroscopically confirmed galaxies, which are detected in radio and mid-IR (MIR) in the redshift range of $0.55 \leq z \leq 1.30$ in the Observations of Redshift Evolution in Large Scale Environments (ORELSE) survey. We constrain the active galactic nuclei (AGN) contribution to the total IR luminosity $\left(f_{\mathrm{AGN}}\right)$, and estimate the AGN luminosity $\left(L_{\mathrm{AGN}}\right)$ and the star formation rate (SFR). Based on the $f_{\mathrm{AGN}}$ and radio luminosity, radio-IR galaxies are split into galaxies that host either high- or low- $f_{\mathrm{AGN}} \mathrm{AGN}$ (high-/low- $f_{\mathrm{AGN}}$ ), and star-forming galaxies (SFGs) with little to no AGN activity. We study the properties of the three radio-IR subsamples comparing to an underlying parent sample. In the comparison of radio luminosity of three sub-samples, no significant difference was found, which could be due to the combined contribution of radio emission from AGN and star formation. We find a positive relationship between $L_{\mathrm{AGN}}$ and specific SFR (sSFR) for both AGN sub-samples, strongly suggesting a coevolution scenario of AGN and SF in these galaxies. A toy model is designed to demonstrate this co-evolution scenario, where we find that, in almost all cases, a rapid quenching time-scale is required, which we argue is a signature of AGN quenching. The environmental preference for intermediate/infall regions of clusters/groups remains across the co-evolution scenario, which suggests that galaxies might be in an orbital motion around the cluster/group during the scenario.
\end{abstract}

Key words: galaxies: active-galaxies: clusters: general-galaxies: evolution-galaxies: star formation - infrared: galaxies - radio continuum: galaxies.

\section{INTRODUCTION}

In the conventional picture of radio galaxies, the dominant source of radio emission is synchrotron radiation from relativistic electrons

*E-mail: lushen@ucdavis.edu

$\dagger$ Visiting Scientist. accelerated by supernova or powered by active galactic nuclei (AGN), with a sub-dominant component of free-free radiation from $\mathrm{H}$ II regions (e.g. Condon 1992). Given the origin of the radio emission, two main populations are detected: star-forming galaxies (SFGs) and galaxies with AGN (see Padovani 2016; Panessa et al. 2019 for recent review). Radio AGN (RAGN) selected by their powerful radio luminosities (i.e. $L_{1.4 \mathrm{GHz}} \gtrsim 10^{24} \mathrm{~W} \mathrm{~Hz}^{-1}$ ) are found to be hosted by red and quiescent galaxies and preferentially reside 
in the cores of clusters, with their AGN powered by inefficient accretion (Best et al. 2005; Tadhunter 2016; Shen et al. 2017). On the other hand, fainter RAGN are found to be hosted by galaxies having ongoing star formation (SF; e.g. Smolčić 2009; Padovani et al. 2011; Hardcastle \& Krause 2013; Gürkan et al. 2015; Rees et al. 2016; Lofthouse et al. 2018; Read et al. 2018). Their AGN are powered by the efficient accretion of cold gas (radiative mode; Ciotti, Ostriker \& Proga 2010; Best \& Heckman 2012; Pierce et al. 2019).

There are considerable observations showing the coeval state of AGN and SF activities of moderate-luminosity AGN selected in Xray (e.g. Lutz et al. 2010; Harrison et al. 2012; Mullaney et al. 2012; Santini et al. 2012; Azadi et al. 2015; Stanley et al. 2015; Scholtz et al. 2018), in radio (e.g. Gürkan et al. 2015), and using various bands (e.g. Lemaux et al. 2014; Cowley et al. 2016). In the simplest interpretation, this coeval nature means the existing cool gas supply on the host galaxy scale can feed the black hole in the centre of the galaxy at the same time as it allows SF in the host galaxy. Major mergers and secular processes (i.e. large galaxy bars and violent disc instabilities) are thought to be responsible for transporting available gas on host galaxy scales to the central regions (e.g. Hopkins \& Quataert 2010; Ellison et al. 2011). However, a question remains on the role of the AGN in regulating the SF activity, either triggering or suppressing it. AGN are known to provide a rapid quenching via radiative winds and large-scale outflows (e.g. Yuan \& Narayan 2014; Gofford et al. 2015; Hopkins et al. 2016), which are more powerful in massive stellar mass hosts (e.g. Kauffmann et al. 2003; Kaviraj et al. 2007; Bongiorno et al. 2016; Lanzuisi et al. 2017). On the other hand, the relationship between AGN power (or black hole accretion rate) and SFR (or SSFR $\equiv \mathrm{SFR} / M_{*}$ ) has shown mixed results (see recent reviews by Alexander \& Hickox 2012; Kormendy \& Ho 2013; Heckman \& Best 2014). Some studies have found a positive correlation (e.g. Netzer 2009; Chen et al. 2013; Gürkan et al. 2015), while others found no trend (e.g. Harrison et al. 2012; Mullaney et al. 2012; Stanley et al. 2015). It has been suggested that these different results could be due to the different variability time-scales of AGN activity and SF (e.g. Hickox et al. 2014). From the perspective of simulations, a comparison of a large sample of X-ray-selected AGN to the EAGLE simulation indicates that the impact of AGN feedback on SF is slow, which can lead to no strong relationship between the specific SFR (sSFR) and instantaneous AGN power (Scholtz et al. 2018).

The environment in which a galaxy resides is also known to trigger and/or quench SF, as shown by the relationships between galaxy colour, morphology, stellar mass, and star formation rate (SFR) with various measures of environment (e.g. Dressler 1980; Peng et al. 2010; Grützbauch et al. 2011; Peng et al. 2012; Lemaux et al. 2019; Tomczak et al. 2019). In simulation studies of cluster environments, it has been found that galaxies follow a 'delayed - then - rapid' quenching scenario in which a galaxy spends a considerable amount of time in a group/cluster environment unquenched, followed by a rapid truncation of its SF (e.g. Wetzel et al. 2013; Muzzin et al. 2014). This scenario is consistent with observational studies in the Observations of Redshift Evolution in Large Scale Environments Survey (ORELSE; Lubin et al. 2009) at $z$ $\sim 1$ using spectroscopically confirmed galaxies in a wide dynamical range covering from cores to outskirts of clusters/groups to the field (Lemaux et al. 2017, 2019; Tomczak et al. 2019), and other studies at similar redshift (e.g. Poggianti et al. 2009; Balogh et al. 2016).

Most of studies on the AGN-SFR relation are focused on Xray-selected AGN hosted in SFGs, which are unbiased with low contamination from AGN and SF activity (Padovani et al. 2017).
Another interesting aspect is to study such relation for radio galaxies when proper classification is applied. In Shen et al. (2017), the properties of radio galaxies and their environmental preferences in large-scale structures (LSSs) at $z \sim 1$ were studied. Radio galaxies were separated into AGN, hybrid, and SFG populations. We found that the hybrid hosts are broadly distributed in colour and stellar mass, with younger stellar ages than the AGN, but older than SFGs. The spectral analyses strongly suggest that they have coeval AGN and SF activity with high accretion efficiency. They do not show clear environmental preferences compared to galaxies of similar colour and stellar mass.

In this work, we continue our study into the coeval nature of AGN and SF activity in radio galaxies and the role of environment. We continue to make use of the ORELSE survey to explore radio galaxies at $z \sim 1$. The ORELSE survey is an extensive photometric and spectroscopic survey of 16 most massive LSSs known at 0.7 $\leq z \leq 1.26$. With hundreds of high-quality spectroscopic redshifts per field, these data make it possible to accurately map out the three-dimensional density field of each LSS, which reveal a full range of environmental densities at these redshifts (see Lemaux et al. 2017; Rumbaugh et al. 2017; Shen et al. 2017; Hung et al. 2019). Taking the advantages of multi-wavelength observations provided by the ORELSE survey, including the combination of midinfrared (MIR) and radio observations, it is possible to separate traditionally selected RAGN and RAGN hosted by SFGs, since it has been found that the former have weak MIR emission or optical obscuration from dust (Whysong \& Antonucci 2004; Ogle, Whysong \& Antonucci 2006). In addition, by including far-infrared (FIR; $\lambda_{\text {obs }}>24 \mu \mathrm{m}$ ) data, one can quantify the SFR in these host galaxies without being biased by AGN-related contamination. Therefore, we use both Very Large Array (VLA) 1.4-GHz imaging and Spitzer/MIPS to locate radio-IR galaxies by matching them to a large data set of spectroscopically confirmed galaxies that are detected in Spitzer/MIPS imaging.

This paper is laid out as follows. An overview of the ORELSE survey and the previously compiled data for this survey, as well as ORELSE observations presented for the first time in this paper, along with a description of the sample selection processes are given in Section 2. In Section 3, we describe the spectral energy distribution (SED) fitting, which estimates the AGN contribution to the total IR luminosity $\left(f_{\mathrm{AGN}}\right)$, AGN IR luminosity $\left(L_{\mathrm{AGN}}\right)$, SFR, and the environment measurements. In Section 4, we compare the properties of radio-IR galaxies in colour, stellar mass, radio luminosity, $L_{\mathrm{AGN}}$ and SFR, and environmental preferences. Our main results regarding the $L_{\mathrm{AGN}}-\mathrm{SFR}$ relationship are shown in Section 5. To explain these observations using a physical picture, we devise a toy model and discuss the interpretation of the coevolution of AGN and SF and the role of environments in Section 6. We conclude with a summary in Section 7. Throughout this paper, all magnitudes, including those in the IR, are presented in the AB system (Oke \& Gunn 1983; Fukugita et al. 1996). We adopt a concordance Lambda cold dark matter $(\Lambda \mathrm{CDM})$ cosmology with $H_{0}=70 \mathrm{~km} \mathrm{~s}^{-1} \mathrm{Mpc}^{-1}, \Omega_{\Lambda}=0.73$, and $\Omega_{\mathrm{M}}=0.27$, and a Chabrier initial mass function (IMF; Chabrier 2003).

\section{DATA AND SAMPLE}

Comprehensive photometric and spectroscopic catalogues of the eight ORELSE fields used in this study have been constructed. These observations span $7 \sim 15 \mathrm{Mpc}$ in the plane of the sky and encompass 32 spectroscopically-confirmed clusters/groups and 97 overdensity candidates, spanning a total mass range of $10^{12.8}$ 
Table 1. The adopted parameters and references of the photometric, spectroscopic, cluster/group catalogues, new overdensity candidates, and local environmental density in the ORELSE survey.

\begin{tabular}{lll}
\hline Catalogues & \multicolumn{1}{c}{ Parameters } & \multicolumn{1}{c}{ References } \\
\hline Photometric & All optical/NIR/MIR photometry, $M_{*}$ & Tomczak et al. (2017, 2019) \\
Spectroscopic & $z_{\text {spec }}$ & Lemaux et al. (2014, 2019) \\
Clusters/groups & Measured centre coordinates, estimated velocity dispersion $\left(\sigma_{v}\right)$ & Methodology presented in Ascaso et al. (2014), \\
& & Hung et al. (2019) \\
New overdensity candidates & Measured centre coordinates, estimated velocity dispersion $\left(\sigma_{v}\right)$ & Hung et al. (2019) \\
Local environmental density & Local overdensity $\left(\log \left(1+\delta_{\text {gal }}\right)\right)$ & Lemaux et al. (2017), Tomczak et al. (2017) \\
\hline
\end{tabular}

to $10^{15.1} \mathrm{M}_{\odot}$. We adopt the available photometric, spectroscopic catalogues, clusters/groups, and new overdensity candidates in the ORELSE survey. We summarize references in Table 1 that describe the fields, the structure properties, the construction of these catalogues, existing data, and parameters adopted in this paper. In this section, we mainly describe new data from the radio and FIR observations in Sections 2.1 and 2.2, respectively. The details of our selection criteria on the radio-IR and spec-IR sample are described in Section 2.3. The spec-IR sample represents an underlying parent sample to isolate radio emission as the only difference. Fig. 1 presents a flowchart summarizing the criteria in defining our specIR sample and three radio-IR sub-samples. We summarize the available fields in Table 2, including their central position, redshift, number of clusters/groups and new overdensity candidates, as well as the number of spectroscopically confirmed and radio galaxies in each field.

In order to probe different structures of AGN and their host galaxies, multi-wavelength observations are necessary (see Padovani et al. 2017 and reference therein). Observations in the near-IR (NIR) to MIR range, especially the latter, probe the dust torus close to the AGN (1-10 pc; e.g. Haas et al. 2008; Stern et al. 2012; Hickox \& Alexander 2018 for recent review). In this paper, we use eight fields (C11350, XLSS005, RX J1053, SG0023, SC1604, RX J0910, RX J1716, and RX J1821) from the ORELSE survey, which have fully reduced radio catalogues and are covered by Spitzer/MIPS imaging, along with accompanying photometric and spectroscopic catalogues. In addition, four fields (XLSS005, RX J1053, SG0023, and RX J0910) have Herschel/SPIRE coverage. We include these observations to improve the estimation of SFR, though we treat galaxies equally regardless of Herschel/SPIRE coverage (see Section 3.1 for more discussion). We note that we do not include Cl1429 in this study due to the poor radio and Spitzer/MIPS imaging quality for this field. We refer the reader to Rumbaugh et al. (2012, 2017), who describe the available fields and LSS properties.

\subsection{Radio observations and catalogues}

All fields were observed using Karl G. Jansky VLA at $1.4 \mathrm{GHz}$ in its B configuration, where the resulting full width at halfmaximum (FWHM) resolution of the synthesized beam is about 5 arcsec and the field of view (i.e. the FWHM of the primary beam) is approximate in 31 arcmin diameter. Net integration times were chosen to result in final $1 \sigma$ sensitivities of about $10 \mu \mathrm{Jy}$ per beam. The details of data reduction and catalogues of the SC1604, SG0023, and RX J1821 obtained from the traditional VLA are described in Shen et al. $(2017,2019)$. Here we describe the details of data reduction and catalogues of the rest of four fields (XLSS005, RX J1053, RX J0910, and RX J1716) obtained from the JVLA.

In general, CASA (Version 4.7.2) was used in calibration and imaging of the VLA data. The standard calibration pipeline was first

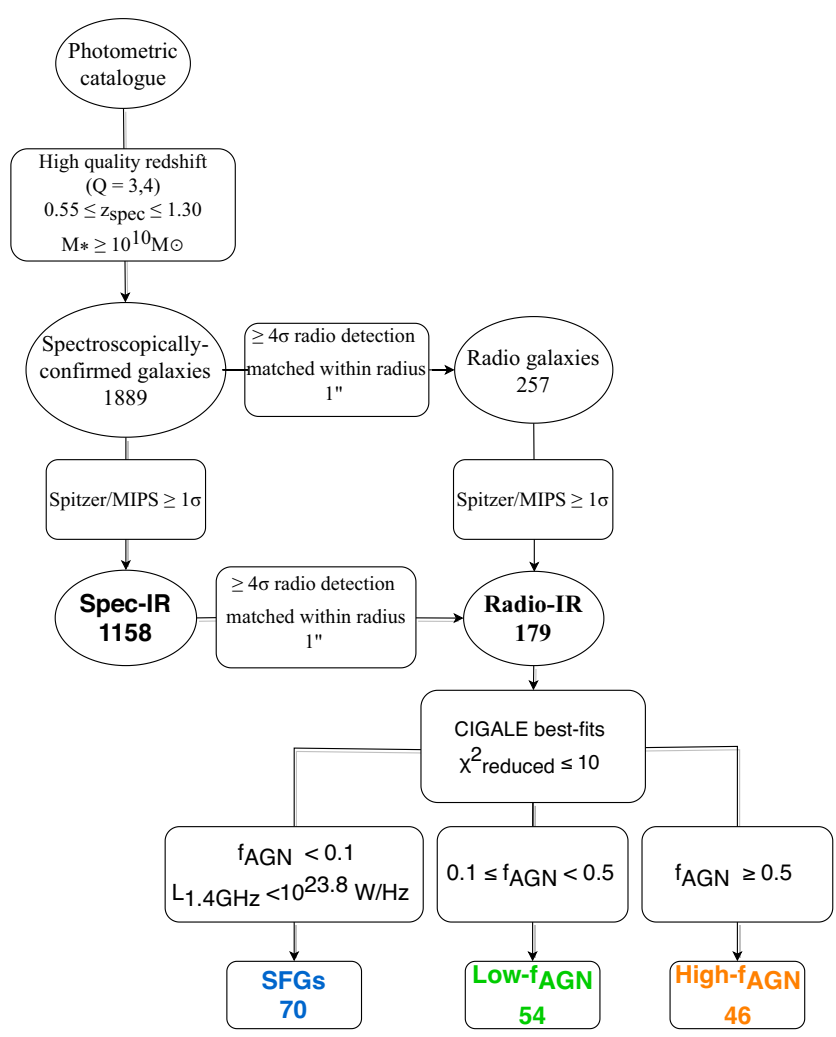

Figure 1. A schematic diagram of our selection process. We started with the full photometric catalogue from Tomczak et al. $(2017,2019)$ and selected those having secure spectroscopic redshifts in the redshift range $0.55 \leq z$ $\leq 1.3$ and $M_{*} \geq 10^{10} \mathrm{M}_{\odot}$ where our spectroscopic catalogue is complete (Shen et al. 2017; Tomczak et al. 2019). To construct a parent sample, we selected spectroscopically confirmed galaxies that are detected $\geq 1 \sigma$ in the Spitzer/MIPS imaging (named as 'spec-IR' sample). The 'radio-IR' galaxies are cross-matched from the Spec-IR sample and VLA 1.4-GHz observations down to a $4 \sigma$ detection. Following the CIGALE (Code Investigating GALaxy Emission) SED best fitting, we use $f_{\mathrm{AGN}}$ and the radio luminosity criteria to split radio-IR galaxies into SFGs, low- $f_{\text {AGN }}$, and high- $f_{\text {AGN }}$ sub-samples. The number of galaxies in each sample are shown in the box. Note that the spec-IR sample includes the radio-IR sample.

run on the $(u, v)$ data for each specific observation data set, where obvious interference and other aberrational data were removed, and the resulting gain and phase calibrations were applied to the target fields. Then automatic flagging of radio frequency interference (RFI) was conducted via the time-frequency crop (TFCrop) and the RFLAG procedures, both of which detect and remove outliers in the 2D time-frequency plane. The target fields were imaged using the TCLEAN algorithm with $w$ projection, which is a widefield imaging technique that takes into account the non-coplanarity 
Table 2. Properties of ORELSE fields and number of spec-IR/radio-IR sample in each field.

\begin{tabular}{|c|c|c|c|c|c|c|c|c|}
\hline Field & $\begin{array}{c}\mathrm{RA}^{a} \\
(\mathrm{~J} 2000)\end{array}$ & $\begin{array}{l}\text { Dec. }^{a} \\
(\mathrm{~J} 2000)\end{array}$ & $\left\langle z_{\text {spec }}\right\rangle^{\mathrm{b}}$ & $\begin{array}{c}\text { Number of } C / G \\
\text { old } / \text { new }^{c}\end{array}$ & $\begin{array}{l}\text { Num. of } \\
\operatorname{spec}^{d}\end{array}$ & $\begin{array}{c}\text { Spec-IR } \\
\text { galaxies }^{e}\end{array}$ & $\begin{array}{c}\text { Radio } \\
\text { galaxies } f\end{array}$ & $\begin{array}{l}\text { Radio-R } \\
\text { galaxies }^{g}\end{array}$ \\
\hline Cl1350 & $13: 50: 48$ & $+60: 07: 07$ & 0.804 & $3 / 3$ & 336 & 198 & 18 & 10 \\
\hline RX J1716 & $17: 16: 50$ & $+67: 08: 30$ & 0.813 & $4 / 5$ & 184 & 97 & 12 & 8 \\
\hline RX J1821 & $18: 21: 38$ & $+68: 27: 52$ & 0.818 & $2 / 0$ & 94 & 46 & 14 & 10 \\
\hline SG0023 & $00: 24: 29$ & $+04: 08: 22$ & 0.845 & $6 / 5$ & 114 & 82 & 8 & 8 \\
\hline SC1604 & 16:04:15 & $+43: 21: 37$ & 0.898 & $10 / 20$ & 649 & 431 & 109 & 75 \\
\hline XLSS005 & $02: 27: 10$ & $-04: 18: 05$ & 1.056 & $1 / 44$ & 314 & 159 & 53 & 27 \\
\hline SC0910 & 09:10:45 & $+54: 22: 09$ & 1.110 & $4 / 17$ & 201 & 138 & 25 & 19 \\
\hline RX J1053 & $10: 53: 40$ & $+57: 35: 18$ & 1.140 & $2 / 3$ & 196 & 121 & 26 & 22 \\
\hline Total & & & 0.920 & $32 / 97$ & 1889 & 1158 & 257 & 179 \\
\hline
\end{tabular}

${ }^{a}$ Coordinates are the centre of radio imaging.

${ }^{b}$ Mean spectroscopic redshift of the main structure in each field. The value in the 'Total' row is the median spectroscopic redshift of the final sample.

${ }^{c}$ The former is the number of clusters and groups that are spectroscopically confirmed using the method presented in Gal et al. (2008); the latter numbers are found by the new VMC technique presented in Hung et al. (2019).

${ }^{d}$ Number of secure spectroscopically confirmed galaxies in the redshift range $0.55 \leq z \leq 1.3$, within $18.5 \leq i^{\prime} / z \leq 24.5$ and

$M_{*} \geq 10^{10} \mathrm{M}_{\odot}$ due to the completeness of our spectroscopic catalogues (see Shen et al. 2017; Tomczak et al. 2019).

${ }^{e}$ Number of galaxies in the spec-IR sample; see the selection in Section 2.3.

${ }^{f}$ Number of radio sources that are matched to the overall spectroscopically confirmed galaxies.

${ }^{g}$ Number of radio galaxies detected at $\geq 1 \sigma$ in Spitzer/MIPS $24 \mu \mathrm{m}$ and have a good fit in CIGALE with reduced $\chi^{2} \leq 10$.

of the baselines as a function of distance from the phase centre. Each $(u, v)$ data set was self-calibrated. The $(u, v)$ data were then concatenated to produce a single $(u, v)$ data file corresponding to the complete set of observations (i.e. combining all observation dates) for each target field. The final rounds of imaging and self-calibration were then performed on these $(u, v)$ data using the same method for each data set.

The final images were then used to generate source catalogues. The NRAO's Astronomical Image Processing System (AIPS) task SAD created the initial catalogues by examining all possible sources having peak flux density greater than three times the local rms noise. We then instructed it to reject all structures for which the Gaussianfitted result had a peak below four times the local rms noise. Because Gaussian fitting works best for unresolved and marginally resolved sources, residual images created by SAD after having subtracted the Gaussian fits from the input images were inspected in order to adjust the catalogue. This step added those extended sources poorly fitted by a Gaussian. Peak flux density, integrated flux density, and their associated flux density errors $(\sigma)$ were generated by SAD. We use the peak flux density unless the integrated flux is larger by more than $3 \sigma$ than the peak flux for each individual source. The depths of radio imaging are shown in Table A1.

To search for optical counterparts to radio sources, we perform a maximum likelihood ratio (LR) technique, following the procedures in section 3.4 of Rumbaugh et al. (2012). In brief, a LR is defined to estimate the excess likelihood that a given optical source is a genuine match to a given radio source relative to a chance alignment. We then carried out a Monte Carlo (MC) simulation to estimate the probability that each optical counterpart is the true match using the LRs. The threshold for matching to a single or double object is the same as that used in Rumbaugh et al. (2012), though in practice, in this paper, only the highest probability matched optical counterpart was considered for each radio source. The optical matching is done to the overall photometric catalogues. We use a search radius of 1 arcsec, aimed at being inclusive, i.e. not to miss any genuine matches due to instrumental/astrometric/astrophysical effects. In this paper, we focus on radio objects that have photometric counterparts with secure spectroscopically-confirmed redshifts with high-quality redshift, within the redshift range $0.55 \leq z \leq 1.3$ and $M_{*} \geq 10^{10} \mathrm{M}_{\odot}$ due to the completeness of our spectroscopic catalogues (see more discussion in Section 2.3). We refer to these galaxies as radio galaxies as shown in Fig. 1. The number of radio galaxies in each field is listed in Table 2.

\subsection{Far-IR data}

In this section, we describe the method of estimating FIR flux from the available Herschel/SPIRE 250-, 350-, and 500- $\mu \mathrm{m}$ imaging. Many different algorithms have been developed to solve the problem of de-blending low-resolution imaging using prior information from high-resolution surveys (Roseboom et al. 2010; Wang et al. 2014; Hurley et al. 2017). In this study, we use the package T-PHOT (Merlin et al. 2015), which implement a maximum likelihood estimation to generate flux density estimates in low-resolution images using galaxy positional priors extracted from a higher-resolution image, to estimate flux density in the Herschel/SPIRE 250-, 350-, and $500-\mu \mathrm{m}$ channels. In general, T-PHOT uses source positions and morphologies of priors from a high-resolution image to obtain photometric analysis of the lower resolution image of the same field. This method was used for measuring galaxy photometry in Spitzer/IRAC and Spitzer/MIPS in this paper by providing real cutouts from the detection image as priors (see Tomczak et al. 2017, 2019 for more details). However, due to the large PSF of SPIRE 250-, 350-, and 500- $\mu \mathrm{m}$ bands images 18, 25, and 37 arcsec, respectively, which is a factor of $>10$ more than our complementary images in short wavelengths, the morphology of priors is not necessary. We, therefore, use source positions as unresolved, pointlike prior to measure the aperture flux of profiles of nearby sources blended together.

In brief, the Herschel/SPIRE level 2.5 point source maps were downloaded from the ESA Herschel Science Archive. A list of priors for positions of possible FIR sources is created using all photometric sources that are detected in Spitzer/MIPS $24 \mu \mathrm{m} \leq 1 \sigma$ and have 'use' flags that reject galaxies having poor photometry (see Tomczak et al. 2017 for details on the 'use' flag). T-PHOT was performed at the positions of the priors for each of the three SPIRE 
images. Due to relatively shallow depth of the MIPS observations, and for completeness for potential sources detected with Herschel that have lower flux at $24 \mu \mathrm{m}$ than our detection limit, we use a $1 \sigma$ detection threshold in $24 \mu \mathrm{m}$. We list in Table A 1 the $3 \sigma / 1 \sigma$ point source completeness limits for the Spitzer/MIPS 24- $\mu$ m images.

To estimate the depth of 250, 350-, and 500- $\mu \mathrm{m}$ SPIRE images, we employ the following procedure. In each image, objects are masked using a segmentation map generated by the Source Extractor software (SEXTRACTOR; Bertin \& Arnouts 1996) with 3 pixels as minimum area (approximately the same size as the beam size of each image) and 1.5 as a significance detection threshold. We measure the fluxes in 1000 randomly placed apertures of diameter $d=4$ pixels (roughly $\sim 1.5$ times the beam size of the images) in empty sky locations. We then fit a Gaussian to the distribution of these fluxes and adopt the standard deviation as an estimate of the $1 \sigma$ detection limit. Across all fields listed in Table A1, we estimate 250-, 350-, and 500- $\mu \mathrm{m}$ imaging depths between $6 \sim 23 \mathrm{mJy}$. We note that the $1 \sigma$ detection limits estimated in this way represent the upper limits in these highly confused images. We also note that by varying the main SEXTRACTOR parameters (i.e. minimum area \pm 1 pixel and detection threshold \pm 0.5 ) would not change the $1 \sigma$ detection limits.

In Appendix A, we show the performance of T-PHOT using simulation of Herschel/SPIRE images. We found that the median of the relative flux difference $\left.\left(f_{\text {measured }}-f_{\text {true }}\right) / f_{\text {true }}\right)$ is close to zero, while the scatter of this quantity increases for low-flux objects. We are confident of the fluxes measured down to $\sim 3$ mJy where the 16 th/84th percentiles of the relative flux difference are $\sim 0.1$, which is lower than the $1 \sigma$ flux density limits in our Herschel/SPIRE $250-\mu \mathrm{m}$ images. See details of this test in Appendix A.

\subsection{Sample selection}

To construct our sample, we started with the full photometric catalogue from Tomczak et al. $(2017,2019)$ and selected those having high-quality spectroscopic redshifts $(Q=3,4$; see Gal et al. 2008; Newman et al. 2013 for the meaning of these values) in the redshift range of $0.55 \leq z \leq 1.3$ and $M_{*} \geq 10^{10} \mathrm{M}_{\odot}$, as shown in Fig. 1. The stellar mass threshold is based on the estimated stellar mass completeness limits for these fields which range between $10^{9}$ and $10^{10} \mathrm{M}_{\odot}$ at these redshifts (see Tomczak et al. 2017). We then select spectroscopically confirmed galaxies that are detected $\geq 1 \sigma$ in the Spitzer/MIPS imaging, named as 'spec-IR' sample. This sample is used as a comparison sample in this study to isolate radio emission as the only difference. The final 'radio-IR' sample is created by cross-matching the spec-IR sample and radio galaxies (see Section 2.1). We obtain a total of 179 radio-IR galaxies. Note that the radio-IR sample is included in the spec-IR sample. The number of galaxies in the spec-IR and the radio-IR samples in each field are listed in Table 2.

As we will describe in detail in Section 3.1, we constrained the AGN contribution $\left(f_{\text {AGN }}\right)$ to the total IR luminosity using the CIGALE SED fitting routine. We further divided the overall radio-IR sample based on the estimated $f_{\mathrm{AGN}}$ and radio luminosity into three subsamples, SFGs, low- and high- $f_{\mathrm{AGN}}$, where SFGs are selected to be SF-dominated galaxies that are best fitted by $f_{\mathrm{AGN}}<0.1$, i.e. with little to no AGN activity. We include an additional radio luminosity threshold to the SFG sample, since SFGs are typically found to have $L_{1.4 \mathrm{GHz}}<10^{23.8} \mathrm{~W} \mathrm{~Hz}^{-1}$ at $z \lesssim 1$ (Padovani 2016; Shen et al. 2017). While our CIGALE results in such cases indicate the lack of an appreciable AGN component, we do not include all types of AGN in the models used for this fitting. To ensure the purity of the SFG sub-sample, we prefer to rely on the high likelihood of a dominant AGN component as evidenced by the high radio luminosity values of these galaxies and exclude them from the analysis. Note that, due to the small numbers, adding these galaxies in the SFG sub-sample would not affect the comparison in Section 4 and the toy model in Section 6.1. Low- and high- $f_{\mathrm{AGN}}$ are selected to be galaxies that host AGN where the AGN contribution in the IR luminosity is high $\left(f_{\mathrm{AGN}} \geq 0.5\right)$ and low $\left(0.1 \leq f_{\mathrm{AGN}}<0.5\right)$. The criteria and the number of galaxies in each sub-sample are shown in Fig. 1.

\section{METHODS}

In this section, we describe the SED fitting that is used to estimate the AGN contribution and SFR in Section 3.1 and the method adopted to estimate local and global environment measurements in Section 3.2.

\subsection{Estimating the AGN contribution and SFR from SED fitting}

We employed the CIGALE (Boquien et al. 2019) in order to constrain the AGN contribution in IR luminosity in a self-consistent framework, considering the energy balance between the UV/optical and IR. We adopt a delayed exponential star formation history (SFH, sfhdelayed) allowing $\tau$ and age to range in similar parameter spaces as those used in Tomczak et al. (2017, 2019). We assume a Chabrier (2003) IMF and the stellar population synthesis models presented by Bruzual \& Charlot (2003) with solar metallicity. Dust attenuation follows Calzetti et al.'s (2000) extinction law allowing colour excess $E(B-V)_{*}$ to vary. The reprocessed IR emission of dust absorbed from UV/optical stellar emission is modelled assuming the dust templates of Dale et al. (2014), allowing the slope $(\alpha)$ to vary in a wide range $\alpha=1 \sim 2.5$ as found in normal SFGs (Dale \& Helou 2002).

For AGN emission, we utilize the models provided in Fritz, Franceschini \& Hatziminaoglou (2006), which take into account two components: the emission of the central source and the radiation from the dusty torus in the vicinity heated by the central source. These models are determined through a set of seven parameters: $R_{\max } / R_{\min }$, the ratio of the maximum to minimum radii of the dust torus; $\tau_{9.7}$, the optical depth at $9.7 \mu \mathrm{m} ; \beta$ and $\gamma$ describing the dust density distribution $\left(\alpha r^{\beta} \mathrm{e}^{\gamma|\cos \theta|}\right)$ with $r$ the radius and $\theta$ the opening angle of the dust torus; $\Phi$, the angle between the AGN axis and the line of sight; and $f_{\mathrm{AGN}}$, the AGN contribution in IR luminosity. In this paper, we decide to fix the values of $R_{\max } / R_{\min }$, $\beta, \gamma, \theta$, which parametrize the density distribution of the dust within the torus to typical values found by Fritz et al. (2006), in order to limit the number of models. We measure the FWHM of the most board emission line (i.e. [O II], [O III], or [Ne III]) in each spectrum of our radio-IR sample (the selection of this sample is in Section 2.3) and adopt a threshold of $>500 \mathrm{~km} \mathrm{~s}^{-1}$ for potential Type 1 AGN. We find a very small fraction (6 per cent) of our radio-IR sample might host Type 1 AGN. Therefore, we fix the $\Phi$ parameter (an angle between the AGN axis and the line of sight) to be $0^{\circ}$, corresponding to a Type 2 AGN (i.e. obscured AGN). We consider a low $\left(\tau_{9.7}=1\right)$ and a high $\left(\tau_{9.7}=6\right)$ optical depth model. The former exhibits a smooth distribution in the MIR, while the latter corresponds to a hidden AGN with a strong silicate absorption (Buat et al. 2015). We consider a full range of AGN fraction $\left(f_{\mathrm{AGN}}\right)$ from 0 to 0.9 , parametrized by the contribution of IR luminosity from AGN to the total IR luminosity (Ciesla et al. 2015). For radio synchrotron emission from either SF or AGN, we consider a correlation coefficient between FIR and radio luminosity 
Table 3. Parameter ranges used in the SED fitting with CIGALE.

\begin{tabular}{|c|c|}
\hline Parameter & Value \\
\hline & sfhdelayed \\
\hline & $0.3,0.5,0.7,1,2,4,6,8,10$ \\
\hline \multirow[t]{2}{*}{ age $[\mathrm{Myr}]$} & $\begin{array}{c}50,100,200,300,400,500,750,1000, \\
3000,5000,8000\end{array}$ \\
\hline & SSP (Bruzual \& Charlot 2003) \\
\hline IMF & 1 \\
\hline \multirow[t]{2}{*}{ Metallicity } & 0.02 \\
\hline & Dust attenuation (Calzetti et al. 2000) \\
\hline$E(B-V)_{*}$ & $\begin{array}{c}0.01,0.05,0.1,0.15,0.2,0.25,0.3 \\
0.4,0.5,0.6,0.8,1.2\end{array}$ \\
\hline \multirow[t]{2}{*}{$E(B-V)_{\text {factor }}$} & 0.44 \\
\hline & Dust emission (Dale et al. 2014) \\
\hline \multirow[t]{2}{*}{$\alpha$} & $0.25,0.5,1.0,1.5,2.0,2.5$ \\
\hline & AGN emission (Fritz et al. 2006) ${ }^{a}$ \\
\hline$R_{\max } / R_{\min }$ & 60.0 \\
\hline$\tau_{9.7 \mu \mathrm{m}}$ & $1.0,6.0$ \\
\hline$\beta$ & -0.5 \\
\hline$\gamma$ & 0.0 \\
\hline$\theta$ & $100^{\circ}$ \\
\hline$\phi$ & 0.001 \\
\hline \multirow[t]{2}{*}{$f_{\mathrm{AGN}}$} & $\begin{array}{c}0.0,0.05,0.1,0.2,0.3 \\
0.4,0.5,0.6,0.7,0.8,0.9\end{array}$ \\
\hline & Radio synchrotron emission \\
\hline$q_{\mathrm{IR}}$ & $1.0,1.5,2.0,2.5,3.0$ \\
\hline$\alpha_{\text {radio }}$ & $0.5,0.7,0.9,1.1$ \\
\hline
\end{tabular}

$\left(q_{\mathrm{IR}}\right)$ taken from the calculated $q_{\mathrm{IR}}$ in our sample, which used the radio luminosities and total IR luminosities estimated by fitting the IR spectral template introduced by Wuyts et al. (2008) to our measured MIPS $24 \mu \mathrm{m}$ photometry. The median calculated $q_{\mathrm{IR}}$ is 2.12 with the scatter spanning from 1.56 to 2.40 as calculated from the $16 \mathrm{th} / 84$ th percentiles. A wide variety of spectral slopes of power-law synchrotron emission $\left(\alpha_{\text {radio }}\right)$ is adopted, including the average spectral slope observed for galaxies hosting a RAGN ( $\alpha=0.7$; i.e. Condon 1992), and extremely steep slope $(\alpha>$ 1) for galaxies hosting obscured $\mathrm{AGN}$ or originating from $\mathrm{SF}$ regions (Ibar et al. 2010). Finally, we adopt the 'pdf_analysis' analysis method in CIGALE to compute the likelihood $\left(\chi^{2}\right)$ for all the possible combinations of parameters and generate the marginalized probability distribution function (PDF) for each parameter and each galaxy (see section 4.3 in Boquien et al. 2019, for full explanation of this method). More details of the parameter settings are shown in Table 3.

We run CIGALE on photometry measured from Spitzer/MIPS $24 \mu \mathrm{m}$, and Herschel/SPIRE 250-, 350-, and 500- $\mu \mathrm{m}$ observations if available, radio $1.4 \mathrm{GHz}$, as well as all available optical and NIR data. An illustration of the data quality and CIGALE SED modelling is shown in Fig. 2. The median $\chi_{\text {reduced }}^{2}$ of the best-fitting SEDs of the radio-IR sample is 2.69 . We define galaxies have $\chi_{\text {reduced }}^{2}>10$ as bad fits, which removes 22 (10 per cent) of the radio-IR targets. After this cut, the median $\chi_{\text {reduced }}^{2}$ is reduced to 2.24 in the final radio-IR sample. In this work, we use SFR, AGN contribution to IR luminosity $\left(f_{\mathrm{AGN}}\right)$ and AGN IR luminosity $\left(L_{\mathrm{AGN}}\right)$ estimated from CIGALE, where $f_{\mathrm{AGN}}$ is defined as $L_{\mathrm{AGN}}=f_{\mathrm{AGN}} \times L_{\mathrm{IR}}$ (Ciesla et al. 2015). To avoid introducing a bias we use the stellar mass estimated from FAST, instead of that estimated from CIGALE, so that we could compare the stellar mass in the radio-IR sample

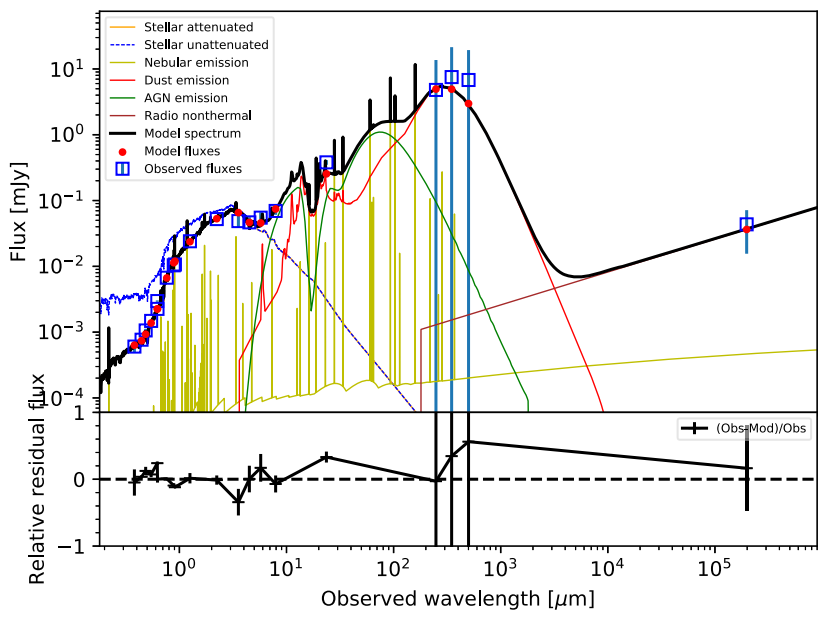

Figure 2. An illustration of the data quality and CIGALE SED modelling. Top panel: the observed photometric fluxes from one radio galaxy $(\mathrm{id}=40319)$ at $z=0.80$ in RX J1053 with errors are shown in blue symbols. The reduced $\chi^{2}$ is 1.71 . The best-fitting CIGALE model is shown in black. Red dots indicate CIGALE-derived photometry in the modelled passbands. The bestfitting CIGALE model is the sum of contributions from an AGN (green dashed line), dust-attenuated stellar emission (orange; the intrinsic stellar emission is indicated in blue), nebular emission (yellow), and dust emission (red). The bottom panel shows the fractional discrepancies between the model and photometry. Note that the best-fitting $f_{\mathrm{AGN}}$ is 0.4 .

to the overall spectroscopically confirmed sample. Moreover, in practice, this choice does not matter, as the difference of stellar mass estimated from FAST and that from CIGALE is negligible. Even when the AGN component is considered, the difference does not change as a function of stellar mass. We show the difference of two estimated stellar masses in the Appendix B. In addition, we further discuss the possible uncertainties and bias of physical properties derived by CIGALE in Appendix B. Due to the large uncertainties of Herschel/SPIRE 250-, 350-, and 500- $\mu$ m photometry, we do not find significant differences when comparing CIGALE best-fitting quantities with or without these data. Thus, we treat galaxies equally regardless of Herschel/SPIRE coverage.

We further ran CIGALE using the same parameter range on the parent spec-IR sample, excluding the radio synchrotron emission templates. We find similar fractions in the spec-IR sample of each sub-sample using the same $f_{\text {AGN }}$ criteria as we used for the radioIR sample. However, galaxies detected in radio are guaranteed to have a starburst and/or AGN component, which is not the case for galaxies in the spec-IR sample. We, therefore, limit our analyses to the radio-IR sample in this paper.

\subsection{Environmental measurements}

Shen et al. (2017) have shown that different types of radio galaxies have different environmental preferences that suggest different scenarios. The AGN are preferentially located in the cores of clusters/groups, suggesting Bondi accretion to be the possible triggering process, while the SFG population exhibits a strong preference for intermediate regions of the clusters/groups, suggesting they could be driven by galaxy-galaxy interactions and merging. Hybrids do not show clear environmental preferences compared to galaxies of similar colour and stellar mass. To further investigate the effect of environment in radio-IR galaxies, we continue to adopt two environment measurements following Shen et al. (2019): a local 
Table 4. Median properties of galaxies in sub-samples, the overall radio-IR, and spec-IR samples.

\begin{tabular}{lcccccccc}
\hline Sample & $z$ & Colour offset & $\log \left(M_{*}\right)$ & $\log \left(1+\delta_{\text {gal }}\right)$ & $\log (\eta)$ & $\log \left(L_{1.4 \mathrm{GHz}}\right)$ & $\log (\mathrm{SFR})$ & $\log \left(L_{\mathrm{AGN}}\right)$ \\
\hline High- $f_{\text {AGN }}$ & $0.95 \pm 0.03$ & $-0.40 \pm 0.04$ & $10.86 \pm 0.03$ & $0.48 \pm 0.04$ & $-0.38 \pm 0.08$ & $23.35 \pm 0.04$ & $0.21 \pm 0.07$ & $11.04 \pm 0.05$ \\
Low- $f_{\text {AGN }}$ & $0.92 \pm 0.02$ & $-0.37 \pm 0.04$ & $10.71 \pm 0.03$ & $0.35 \pm 0.03$ & $-0.61 \pm 0.09$ & $23.43 \pm 0.03$ & $0.77 \pm 0.04$ & $10.40 \pm 0.05$ \\
SFGs & $0.90 \pm 0.01$ & $-0.67 \pm 0.06$ & $10.65 \pm 0.04$ & $0.47 \pm 0.03$ & $-0.45 \pm 0.07$ & $23.30 \pm 0.02$ & $1.02 \pm 0.05$ & - \\
Radio-IR $^{a}$ & $0.92 \pm 0.01$ & $-0.47 \pm 0.02$ & $10.73 \pm 0.02$ & $0.45 \pm 0.02$ & $-0.48 \pm 0.04$ & $23.36 \pm 0.02$ & $0.77 \pm 0.04$ & - \\
Spec-IR & $0.90 \pm 0.003$ & $-0.42 \pm 0.01$ & $10.58 \pm 0.01$ & $0.37 \pm 0.01$ & $-0.43 \pm 0.02$ & - & - & - \\
\hline
\end{tabular}

Note that radio-IR galaxies with $f_{\mathrm{AGN}}<0.1$ and $L_{1.4 \mathrm{GHz}}>10^{23} \mathrm{~W} \mathrm{~Hz}^{-1}$ are not included in any of the above three sub-samples. ${ }^{a}$ The overall radio-IR galaxies.

environment that probes the current density field to which a galaxy is subject and a global environment that probes the time-averaged galaxy density to which a galaxy has been exposed.

We adopt the $\log \left(1+\delta_{\text {gal }}\right)$ as local environment measurement obtained using a Voronoi Monte Carlo (VMC) algorithm which is described in full detail in Lemaux et al. (2017) and Tomczak et al. (2017). We adopt $\eta=R_{\text {proj }} / R_{200} \times|\Delta v| / \sigma_{v}$ as the global environment measurement following the method described in Shen et al. (2017). The final cluster/group catalogue is derived from the previous spectroscopically confirmed clusters and groups combined with new overdensity candidates. In the former case, clusters and groups are spectroscopically confirmed using the method presented in Gal et al. (2008). The cluster centres are obtained following the methodology of $i$-luminosity-weighted centre of the members galaxies as described in Ascaso et al. (2014). In the latter case, new overdensity candidates are found using local overdensity maps, down to total overdensity masses of $\log \left(M_{\text {tot }} / \mathrm{M}_{\odot}\right)>13.5$ (Hung et al. 2019). The $\sigma_{v}$ of these new structures are velocity dispersions calculated from $\log \left(M_{\text {tot }}\right)$ according to equations (1) and (2) in Lemaux et al. (2012). In this paper, we separate galaxies to be in the clusters/groups core $(\eta \leq 0.1)$, intermediate region $(0.1<\eta \leq$ $0.4)$, infall region $(0.4<\eta \leq 2)$, and field region $(\eta>2)$.

\section{GALAXY PROPERTIES OF RADIO-IR SUB-SAMPLES}

Radio galaxies detected in the MIR are divided into SFGs, lowand high- $\mathrm{f}_{\mathrm{AGN}}$ sub-samples as described in Section 2.3. In this section, we explore differences among the three radio-IR galaxy sub-samples both in comparing them between each other and as compared to an underlying parent sample, in terms of their optical colour, stellar mass, redshift, and environments. The spec-IR sample that contains the radio-IR sample is used as the underlying parent galaxy population. In addition, in the environmental analyses, we compare the three radio-IR galaxy sub-samples to their carefully designed control samples, in order to exclude the effect of colour and stellar mass on the environmental preference. These control samples are constructed from the spec-IR sample, after excluding all radio-IR galaxies, and is matched on the colours and stellar mass of each radio-IR sub-sample. We then present internal comparisons of radio luminosity, SFR, AGN luminosity $\left(L_{\mathrm{AGN}}\right)$ in IR between each radio-IR sub-sample, which imply a correlation between SFR and AGN luminosity. Median values of these properties for each sub-sample, radio-IR, and spec-IR are listed in Table 4.

\subsection{Radio galaxy colours}

It has been found that radio galaxies occupy the full range of colour from blue SFGs to galaxies, which host traditionally selected RAGN that are red and quiescent, while IR-detected galaxies are predominantly in the star-forming region. Here, we apply a two- colour selection technique proposed by Williams et al. (2009) to divide the galaxies into two categories: quiescent and star forming galaxies. We adopt the rest frame of $M_{\mathrm{NUV}}-M_{\mathrm{r}}$ versus $M_{\mathrm{r}}-$ $M_{\mathrm{J}}$ colour-colour diagram following separation lines from Lemaux et al. (2014). Specifically, the line is defined as that region within $M_{\mathrm{NUV}}-M_{\mathrm{r}}>2.8\left(M_{\mathrm{r}}-M_{\mathrm{J}}\right)+1.51$ and $M_{\mathrm{NUV}}-M_{\mathrm{r}}>3.75$ at $0.55 \leq z \leq 1.0$ and region within $M_{\mathrm{NUV}}-M_{\mathrm{r}}>2.8\left(M_{\mathrm{r}}-M_{\mathrm{J}}\right)$ +1.36 and $M_{\mathrm{NUV}}-\mathrm{M}_{\mathrm{r}}>3.6$ at $1.0<z \leq 1.3$ are considered quiescent. We then calculate a 'colour offset' value for galaxies as their perpendicular offset to quiescent and star-forming separation lines according to their $z_{\text {spec }}$, with positive representing galaxies in the quiescent region.

In panel (a) of Fig. 3, we show the colour offset histograms for the spec-IR and the three radio-IR sub-samples. The median values are marked as vertical lines and shaded by $1 \sigma$ uncertainty. Uncertainties on the median colour offset are given by $\sigma_{\mathrm{NMAD}} / \sqrt{n-1}$, where $\sigma_{\mathrm{NMAD}}$ is the normalized median of the absolute deviations (Hoaglin, Mosteller \& Tukey 1983) and $n$ is the number of the sample (see Shen et al. 2019). Throughout this paper, we conservatively adopt $\sigma_{\mathrm{NMAD}} / \sqrt{n-1}$ as the formal uncertainty. It appears that the median colour offset of SFGs is clearly separated from others, with their colour offset histogram extending to lower values.

We employed the Kolmogorov-Smirnov statistic (K-S) test and the resultant $p_{\mathrm{ks}}$ value $^{1}$ to test whether the colour offset distributions of three sub-samples and the overall radio-IR/spec-IR samples are consistent with being draw from the same distribution. The $p_{\mathrm{ks}}$ values on the SFG sample are 0.03 and $\approx 0$, which confirm that SFGs do not share the same colour offset distribution as the radioIR and spec-IR samples. For low- and high- $f_{\text {AGN }}$, the median values largely overlapped with each other and that of the spec-IR sample, with differences between high- $f_{\mathrm{AGN}}$ and spec-IR distribution found in the K-S test. The AGN sub-samples are, on average, closer to the quiescent region, indicating their stellar populations are older than that of SFGs. To test this point, we take the age of the main stellar population in the galaxy ('sfh.age_main' parameter) estimated in CIGALE, which represents the time since the onset of SF and then following a delayed SFH. The average age of SFGs is $\sim 2 \mathrm{Gyr}$, while that of the two AGN sub-samples is $\sim 3$ Gyr.

Overall, MIR-detected galaxies dominate in the star-forming region (i.e. colour offset $<0$ ). 26 per cent of spec-IR are quiescent galaxies, compared to 40 percent for the overall spectroscopy galaxies in the same redshift and stellar mass range in the ORELSE survey (Lemaux et al. 2019). The fractions of quiescent galaxies are even smaller, $\sim 10$ per cent for the three radio-IR sub-samples. AGN are predominantly hosted by galaxies in the star-forming region,

\footnotetext{
${ }^{1}$ In this paper, we adopt the K-S test and the resultant $p_{\mathrm{ks}}$ value that if $p_{\mathrm{ks}}$ $>0.05$, we cannot reject the hypothesis that the two distributions are drawn from the same distribution. Otherwise, we say the probability of drawing from the same distribution is very small.
} 

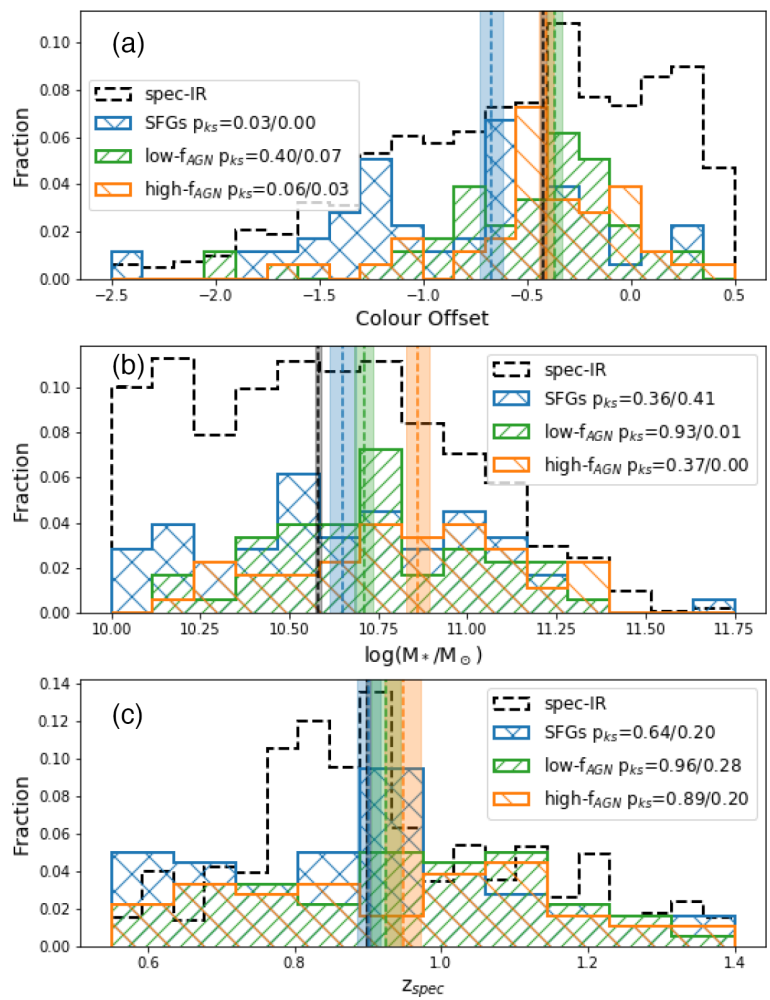

Figure 3. Panel (a): colour offset histograms of spec-IR (dashed black) and three radio-IR high- $f_{\text {AGN }}$ (orange), low- $f_{\text {AGN }}$ (green), and SFG (blue) subsamples, scaled by the total number of radio-IR samples. A positive colour offset value represents galaxies in the quiescent region, see Section 4.1. Panel (b): stellar mass histograms of spec-IR and three radio-IR subsamples. Panel (c): $z_{\text {spec }}$ histogram of spec-IR and three radio-IR subsamples. Median values of each sample are shown in dashed vertical lines and $1 \sigma$ uncertainties are shown as shaded regions with the same colours as used in the histograms. The $p$-value result of the K-S test $\left(p_{\mathrm{ks}}\right)$ between each radio-IR sub-sample and the overall radio-IR/spec-IR sample are shown in the legend after labels. For a reference, if $p_{\mathrm{ks}}<0.05$, the two distributions are not drawn from the same distribution.

which is in line with studies that show IR-detected RAGN are not hosted by red and quiescent galaxies (e.g. Magliocchetti et al. 2018) and other studies of obscured AGN (e.g. Chang et al. 2017).

\subsection{Stellar mass}

The stellar mass histograms are shown in panel (b) of Fig. 3. We find that all radio-IR sub-samples are hosted by more massive galaxies than the overall spec-IR sample at $\gtrsim 3 \sigma$ level. This result is consistent with studies showing that the probability for a galaxy to be a radio source increases with increasing stellar mass (e.g. Ledlow \& Owen 1996). Furthermore, it seems that low- and high$f_{\text {AGN }}$ hosts are more massive than SFGs. Specifically, we find that high- $f_{\mathrm{AGN}}$ hosts skew toward higher stellar masses among the three radio sub-samples. We apply $\mathrm{K}-\mathrm{S}$ tests to confirm this difference. The $p_{\mathrm{ks}} \mathrm{s}$ between low-/high- $f_{\mathrm{AGN}}$ and spec-IR are small, implying they are not drawn from the same distribution. The K-S tests is not conclusive between the SFG sub-sample and the overall spec-IR sample. Again, as compared to traditionally selected RAGN hosts, at $z \sim 1$ and the same stellar mass limit, the median stellar mass is found to be $10^{11.16}$ (Shen et al. 2017). Neither high- nor low$f_{\text {AGN }}$ reach this median value. It is likely that AGN selected in this study are not hosted by the same type of galaxy hosting traditionally selected RAGN are massive and dominate the quiescent region.

\subsection{Redshift}

In panel (c) of Fig. 3, we show the redshift distribution of the three radio-IR sub-samples, as well as the spec-IR sample. The median $z_{\text {spec }}$ s largely overlap with each other. None of the K-S tests conclusively confirm any difference between the sub-samples and the overall radio-IR and spec-IR sample. Therefore, these results suggest that the three radio-IR sub-samples are similar.

\subsection{Environments}

RAGN are preferentially found in the cores of galaxy clusters and locally overdense environments both in the local universe (e.g. Miller \& Owen 2002; Best 2004) and at high redshifts $(z>0.5$; e.g. Magliocchetti et al. 2004; Best et al. 2014; Lindsay et al. 2014). However, RAGN detected in MIR are preferentially found in the field at $z \leq 1.2$ (Magliocchetti et al. 2018). On the other hand, SFGs are broadly distributed in terms of local overdensity (Tomczak et al. 2019). Therefore, it is imperative to explore the role of both the clusters/groups and local environment on the three radio-IR subsamples.

We plot the cumulative distribution functions (CDFs) of the $\log \left(1+\delta_{\text {gal }}\right)$ of each radio-IR sub-sample, as well as the overall trend of the spec-IR sample in the top left-hand panel of Fig. 4. We find that the median $\log \left(1+\delta_{\text {gal }}\right)$ of the low $-f_{\text {AGN }}$ galaxies overlaps with the median value of the spec-IR sample, being lower than that of SFGs and high- $f_{\mathrm{AGN}}$ galaxies. The latter two sub-samples share similar median values. To confirm a local environmental effect, rather than those due to galaxy type and stellar mass that can bias the analyses of environments, we draw 100 control samples from the spec-IR sample, excluding radio galaxies, matching each radioIR sub-sample on colour and stellar mass. In brief, a control sample is identified using a 3D matching algorithm, following the Shen et al.'s (2017) method, which ensures that the distributions of $M_{*}$ and two rest-frame colours $\left(M_{\mathrm{NUV}}-M_{\mathrm{r}}, M_{\mathrm{r}}-M_{\mathrm{J}}\right)$ of the control sample match closely to those of the parent radio sub-samples. K$\mathrm{S}$ tests are run on the $\log (\eta)$ and $\log \left(1+\delta_{\text {gal }}\right)$ distributions of the parent and control sample. In order to explore the full breadth of possible outcomes for this comparison, we perform 100 iterations of control sampling and K-S testing. The mode of the $p_{\mathrm{ks}}$ is obtained by binning $p_{\mathrm{ks}} \mathrm{s}$ into 10 bins and returning the bin with the most $p_{\mathrm{ks}}$. In the right-hand panels of Fig. 4, we show the local overdensity CDF of each radio-IR sub-sample and median CDF of its control samples, along with a $1 \sigma$ (i.e. the 16 and 84 per cent values) shaded region. The left- to right-hand panels correspond to SFG, low-, and high $-f_{\mathrm{AGN}}$ comparison. The modes of the $p_{\mathrm{ks}} \mathrm{s}$ are shown in the panels. The SFG-control sample appears slightly different from the SFG population in the less dense regions, which is confirmed by the small mode of the $p_{\mathrm{ks}}$. This result indicates that SFGs have a preference to be in locally denser environments than its control sample. The CDF of the low- $f_{\mathrm{AGN}}$ galaxies shows an enhancement in the intermediate density region compared to its control sample. The mode of $p_{\mathrm{ks}}$ is 0.06 , which is close to the significance threshold of the K-S test. This result might hint that the local environmental preference of low- $f_{\mathrm{AGN}}$ is the intermediate density region. Even though there are small differences in the high- $f_{\text {AGN }}$ panels, the K-S tests are not conclusive. We will further interpret these results when combining the results from the global environment. 

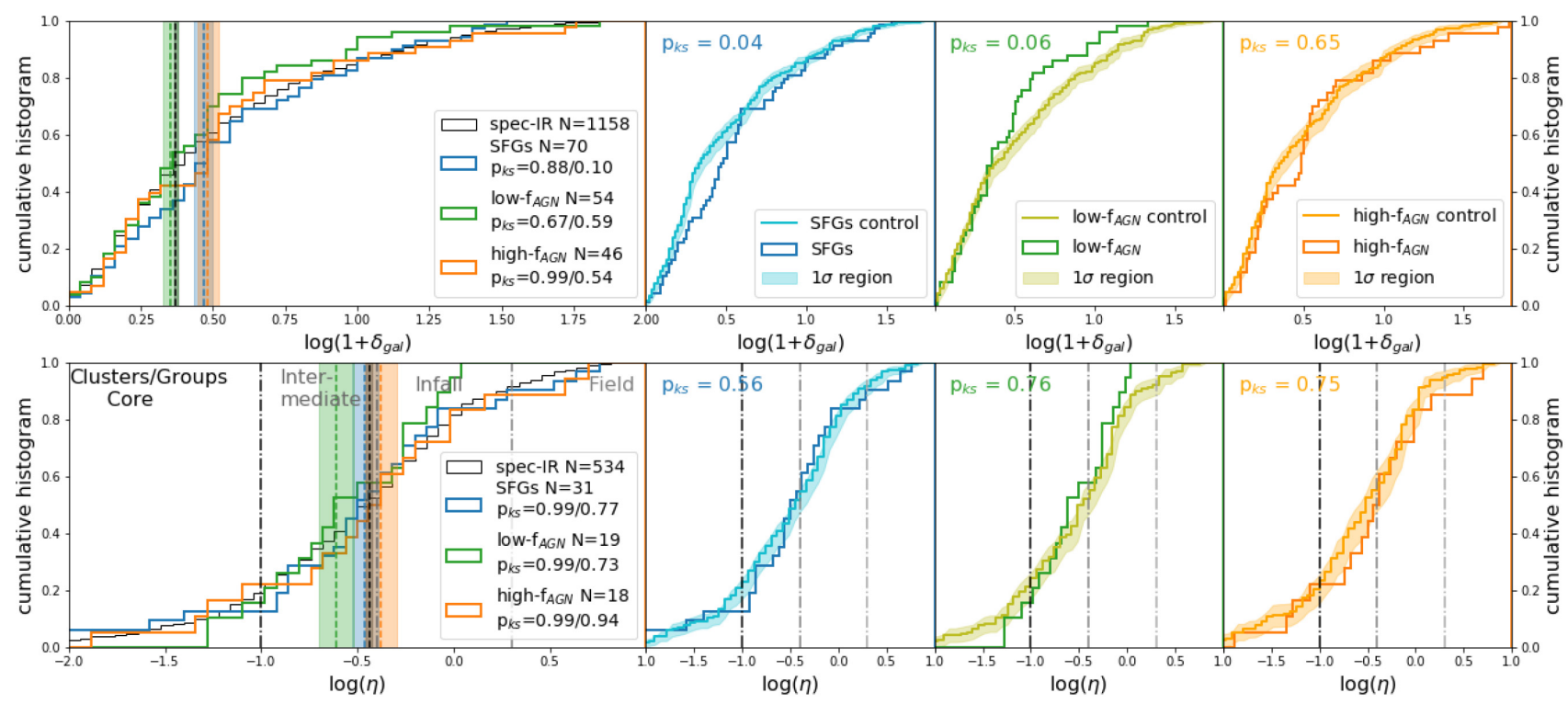

Figure 4. Local and global environment CDFs of the three radio-IR sub-samples and spec-IR sample. Top left-hand panels: $\mathrm{CDFs}$ of log $\left(1+\delta_{\text {gal }}\right)$ of high$f_{\mathrm{AGN}}$ (orange line), low- $f_{\mathrm{AGN}}$ (green line), and SFGs (blue line) and spec-IR (black dashed line). The median values are shown in coloured dashed vertical line with shaded region corresponding to $1 \sigma$ uncertainties. The number of galaxies in each sample in both plots is displayed after their label, as well as the $p_{\mathrm{ks}}$ values of the K-S test between each sub-sample and the overall radio-IR/spec-IR sample. Top right-hand panels: from the left to right, corresponding to comparisons of local $\log \left(1+\delta_{\text {gal }}\right)$ CDFs of SFGs, low-, high- $f_{\text {AGN }}$ sub-sample. The lines with shaded regions are median values and the $1 \sigma$ spread (i.e. the 16 and 84 per cent values) of the 100 control samples. The mode of $p_{\text {ks }}$ are presented in each panel. Bottom panels: CDFs of $\log (\eta)$ of high- $f_{\text {AGN }}$ (orange line), low- $f_{\mathrm{AGN}}$ (green line), SFGs (blue line), and spec-IR (black dashed line), limited to galaxies having $R_{\mathrm{proj}} / R_{200}<3$ and $\left|\delta v / \sigma_{v}\right|<3$. This cut limit galaxies to be associated with an individual cluster/group. For a reference, three dashed lines of constant $\log (\eta)$ are displayed to separate between clusters/groups core ( $\eta$ $\leq 0.1)$, intermediate $(0.1<\eta \leq 0.4)$, infall regions $(0.4<\eta \leq 2)$, and field region $(\eta>2)$. Bottom right-hand panels: from the left- to right-hand side, CDFs of $\log (\eta)$ of SFGs, low- and high- $f_{\mathrm{AGN}}$, and median values and $1 \sigma$ spreads (i.e. the 16 and 84 per cent values) of the 100 control samples. Three lines of constant $\log (\eta)=0.1,0.4$, and 2.0 are displayed as well.

With the recently updated clusters/groups catalogues in the ORELSE fields (Hung et al. 2019), we are able to measure the global environment in a more consistent way. We found that 40 percent of the SFGs are in the cluster/group environment (i.e. $\log (\eta) \leq$ 2) versus 32 percent of galaxies in the low- $f_{\text {AGN }}$ galaxies and 44 percent in the high- $f_{\text {AGN }}$. To obtain a more quantitative look at the environment distribution within clusters/groups, we plot the $\log (\eta)$ CDFs of each sub-sample as well as the overall trend of spec-IR sample in the bottom panels of Fig. 4. Note that we adopt the cut of $R_{\text {proj }} / R_{200} \leq 3$ and $\left|\delta v / \sigma_{v}\right| \leq 3$ to include galaxies that are clearly associated with an individual cluster/group. The number of galaxies in each sub-sample after applying this cut are shown in the legend. It appears that the median values of the three radioIR cluster/group sub-samples and the spec-IR cluster/group sample largely overlap, with their median values in the intermediate and infall regions. We find that low $-f_{\mathrm{AGN}}$, on average, have marginally $\sim 2 \sigma$ lower $\log (\eta)$ values than that of SFGs and high- $f_{\text {AGN }}$, which seems like an opposite behaviour as shown in local overdensity distributions. It might due to the difference of sample selections, i.e. galaxies associated with clusters/groups or field galaxies. The median $\log (\eta)$ of the overall SFG, low-, and high- $f_{\text {AGN }}$ sub-samples are $0.71,1.11$, and 0.73 , respectively, which is consistent with the local overdensity preferences. Note that a $\operatorname{larger} \log (\eta)$ means the galaxy resides further away from the cluster/group cores. The relatively large median $\log (\eta)$ value of the overall low- $f_{\mathrm{AGN}}$ subsample suggests that there are more field galaxies. We further test if the local density preferences is due to galaxies associated with clusters/groups or in field. We find that the median $\log \left(1+\delta_{\text {gal }}\right)$ of clusters/groups galaxies in the low- $f_{\text {AGN }}$ sub-samples is $0.61 \pm 0.06$, which is lower than that of other two sub-samples $(0.68 \pm 0.06$ for high- $f_{\mathrm{AGN}}$ and $0.79 \pm 0.04$ for SFGs), while the median $\log \left(1+\delta_{\text {gal }}\right)$ values of field galaxies in the three sub-samples overlaps within their uncertainties. These might suggest that it may be clusters/groups galaxies in low- $f_{\text {AGN }}$ that possibly assert themselves to give the local environmental preference. None of the K-S tests on the distribution of $\log (\eta)$ of the three radio-IR clusters/groups sub-samples and the radio-IR/spec-IR clusters/groups sample are conclusive as shown in the legends after the labels.

We again run the control sample comparison as applied in the analyses of local environment to investigate more carefully the cluster/group preference. Note that here we draw control samples from the spec-IR sample within $R_{\text {proj }} / R_{200} \leq 3$ and $\left|\delta v / \sigma_{v}\right| \leq 3$, matching galaxies within this phase space in each radio-IR subsample. As shown in the three bottom right-hand panels of Fig. 4, the $\log (\eta)$ distributions of the controls largely overlap with their parent radio-IR populations. These results suggest that radio-IR subsamples do not have any preference in terms of cluster/group environments compared to the overall spec-IR sample and colour-stellar mass-matched control samples. However, we note that the number of galaxies in each sub-sample is small, i.e. only $\sim 20$ galaxies in each, which might obscure differences between these populations.

Combining the results from the local and global environments, the SFG population marginally tends to be located in locally dense regions, corresponding to the intermediate/infall regions of clusters/groups, with such local environmental preference persisting when comparing to its colour-stellar mass control sample. The properties of the SFG population in this paper are largely consistent with the overall radio-selected SFG population found in the five 
ORELSE LSSs (Shen et al. 2017), in terms of colour, local, and global environment, though a higher stellar mass on average is seen in the LSSs. We note that although the two studies use the same stellar mass cut and are at comparable radio depth, different classification methods are adopted, which might lead to the small differences in the stellar mass.

The low- $f_{\text {AGN }}$ sub-sample tends to be located in locally intermediate density regions, corresponding to the intermediate/infall regions of clusters/groups, with such local environmental preference persisting when comparing to its colour-stellar mass control sample. The high- $f_{\text {AGN }}$ sub-sample shares the same environmental preference both locally and globally as their colour-stellar mass control samples. Both AGN sub-samples avoid the core of clusters/groups where traditionally selected RAGN are preferentially to be located (Hickox et al. 2009; Shen et al. 2017). These results are consistent with other studies of radio galaxies. Bardelli et al. (2010) found that RAGN hosted by SFGs and pure SFGs at $0<z$ $<1$ do not show significantly different environmental distributions compared to control samples with similar IR colour and sSFR, while traditionally selected RAGN show a significant preference to be in denser regions. Magliocchetti et al. (2018) showed that RAGN that emit at MIR have a significant preference to be in the less dense field-like environment, compared to RAGN that are not detected in MIR.

\subsection{Radio luminosity}

In studies of RAGN, radio luminosity is seen to strongly correlate with the black hole mass $\left(\mathrm{M}_{\mathrm{BH}}\right)$ and anti-correlate with Eddington ratio $\left(\lambda_{\mathrm{Edd}}=L_{\mathrm{bol}} / L_{\mathrm{Edd}}\right)$, using a variety of AGN classification methods (e.g. Laor 2000; Ho 2002; McLure \& Jarvis 2004; Sikora, Stawarz \& Lasota 2007; Chiaberge \& Marconi 2011; Sikora \& Begelman 2013; Ishibashi et al. 2014). On the other hand, thanks to the well-established FIR-radio correlation (Condon 1992; Kennicutt 1998; Yun, Reddy \& Condon 2001), radio luminosity is a good indicator of the SFR for SFGs (Bell et al. 2003; Hopkins et al. 2003).

In panel (a) of Fig. 5, we show the histograms of radio luminosity $\left(L_{1.4 \mathrm{GHz}}\right)$ of the three radio-IR sub-samples. The three histograms are not statistically identical based on the large $p$-value from the $\mathrm{K}-\mathrm{S}$ test. The low- $f_{\mathrm{AGN}}$ sub-sample has, on average, slightly higher radio power than the high- $f_{\mathrm{AGN}}$ sub-sample at $2 \sigma$ and SFGs at $3 \sigma$ significant level. There are seven galaxies having $L_{1.4 \mathrm{GHz}} \geq 10^{23.8} \mathrm{~W}$ $\mathrm{Hz}^{-1}$, six in low- $f_{\mathrm{AGN}}$, and one in high- $f_{\mathrm{AGN}}$. This radio luminosity threshold is the typical radio luminosity cut for RAGN at $z \sim 1$ by other studies (e.g. Hickox et al. 2009; Shen et al. 2017, 2019). Note that we apply this radio power threshold when selecting the SFGs population to exclude AGN in this sub-sample but not in the lowand high- $f_{\text {AGN }}$ sub-samples (see Section 2.3 for more details on this radio power threshold).

\subsection{Star formation rate}

In panel (b) of Fig. 5, we show the histograms of SFR derived in CIGALE of the three radio-IR sub-samples. It appears that SFGs have, on average, the highest SFR, then the low- $f_{\mathrm{AGN}}$, while the high $-f_{\text {AGN }}$ extend to lower values. The median radio luminosity for our SFGs is $\log \left(L_{1.4 \mathrm{GHz}}\right)=23.30$, corresponding to $\mathrm{SFR}=66.38 \mathrm{M}_{\odot} \mathrm{yr}^{-1}$, using the SFR formula from $1.4 \mathrm{GHz}$ from Bell et al. (2003) and converting Salpter IMF into Charbier IMF by multiplying by a factor of 0.6 . However, the median SFR derived from CIGALE is $10.56 \mathrm{M}_{\odot} \mathrm{yr}^{-1}$. There are two reasons for
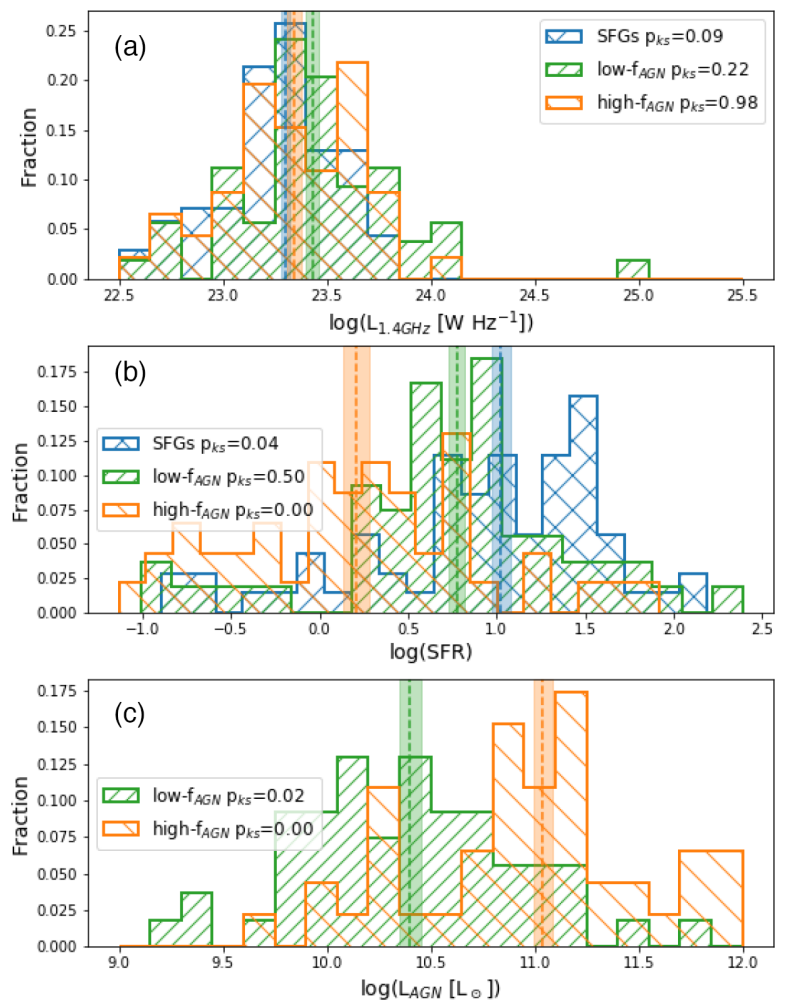

Figure 5. Panel (a): $L_{1.4 \mathrm{GHz}}$ histograms of three radio-IR sub-samples. Panel (b): $\log$ (SFR) histograms of three radio-IR sub-samples. Panel (c): $L_{\mathrm{AGN}}$ histograms of three radio-IR sub-samples. Median and $1 \sigma$ uncertainty values of each sample are shown in dashed vertical line and shaded region with the same colours as used in the histograms. Uncertainties on $L_{1.4 \mathrm{GHz}}$, $\log (\mathrm{SFR}), L_{\mathrm{AGN}}$ of sample are the $\sigma_{\mathrm{NMAD}} / \sqrt{n-1}$. The $p$-value result of the K-S test $\left(p_{\mathrm{ks}}\right)$ between the three radio-IR sub-samples to the overall radio-IR are shown in each panel after the label. In the bottom panel, the $p_{\mathrm{ks}} \mathrm{S}$ are the K-S test results between the high-/low- $f_{\mathrm{AGN}}$ sub-samples to the combined high- and low- $f_{\mathrm{AGN}}$ sample. For a reference, if the $p_{\mathrm{ks}}<0.05$, the two distributions are not drawn from the same distribution.

this discrepancy. One reason is that a rapid, strong episode of SF could produce long-lived stars that contaminate the bands tracing SF for hundreds of Myr afterwards. This contribution is corrected for CIGALE, which, in turn, lowers the SFR derived in this method (Boquien, Buat \& Perret 2014; Boquien et al. 2016). An other reason is that SFR estimators calibrated on long time-scales are valid for galaxies with long, gradual SF episodes but not for starbursting galaxies. Therefore, assuming a single exponential decaying SFH might underestimate the SFR for these SFGs as they are in a starbursting episode.

\subsection{AGN power}

In panel (c) of Fig. 5, we show the histograms of AGN IR luminosity $\left(L_{\mathrm{AGN}}\right)$ of the high- and low- $f_{\mathrm{AGN}}$ sub-samples (see Section 3.1 for the definition of $L_{\mathrm{AGN}}$ ). We find a clear offset in both median values and their histograms. The K-S tests on the $L_{\mathrm{AGN}}$ distribution of high $-f_{\text {AGN }}$ and low $-f_{\text {AGN }}$ is $<0.02$, confirming the difference in $L_{\mathrm{AGN}}$ distributions. In the radio luminosity comparison, we found a marginally higher, on average, radio luminosity for low- $f_{\mathrm{AGN}}$. These results seem inconsistent with the general picture of AGN luminosity increasing with increasing radio luminosity (Sikora \& Begelman 2013; Ishibashi et al. 2014), since the bulk of the energy 
is released from AGN in a kinetic form via radio jets (Churazov et al. 2005). However, these studies are mainly focused on the powerful RAGN $L_{1.4 \mathrm{GHz}}>10^{25}$, much higher than the radio luminosity of our sample.

In fact, studies of radio sources suggest the origins of radio luminosity from AGN accretion (Zakamska et al. 2016; White et al. 2015, 2017) and/or SF activities (e.g. Kimball et al. 2011; Condon et al. 2013). In order to see if a combination of mechanisms could explain our result, we apply a simple test. From the AGN side, following a radio luminosity and X-ray luminosity correlation with a slope of $1.2 \pm 0.15$ (Panessa et al. 2015) calibrated for AGN at high Eddington ratios (i.e. $\left.\left(L_{\mathrm{AGN}} / L_{\mathrm{Edd}}\right)>10^{-3}\right)$, we obtain $\Delta \log L_{1.4 \mathrm{GHz}}=1.2 \Delta \log L_{\mathrm{X} \text {-ray }}$. We then assume that $\mathrm{AGN}$ bolometric luminosity $L_{\mathrm{AGN}} \propto L_{\mathrm{X} \text {-ray }}$ with a constant correction factor (Brightman et al. 2017); thus, $\Delta \log L_{1.4 \mathrm{GHz}}=1.2 \Delta \log L_{\mathrm{AGN}}$. We apply this correlation to the observed difference in $L_{\mathrm{AGN}}$, assuming that for Type 2 AGN $L_{\mathrm{AGN}}$ mostly contributes to the total IR range. The median $L_{\mathrm{AGN}}$ of high- $f_{\mathrm{AGN}}$ galaxies is $0.6 \mathrm{dex}$ higher than that of low- $f_{\text {AGN }}$ galaxies, corresponding to $\sim 0.5 \mathrm{dex}$ higher in radio luminosity. From the SFR side, the median SFR of high- $f_{\mathrm{AGN}}$ galaxies is 0.5 dex lower than that of low- $f_{\mathrm{AGN}}$ galaxies, following the radio luminosity-SFR correlation (Bell et al. 2003), corresponding to the same difference in radio luminosity. Thus, the confluence of the radio emission generated by the two mechanisms could lead to the similar median values of radio luminosity of highand low- $f_{\text {AGN }}$ sub-samples. Note that it is not necessary to include different weights on the contribution of AGN versus SF, as these have been included in the differences of the observed $L_{\mathrm{AGN}}$ and SFR, and the conversion between these two contributions and radio luminosity are of the same order.

\subsection{Summary of galaxy properties in the radio-IR sub-samples}

As a summary for the analyses of this point, the overall radio-IR galaxies are situated in the dusty star-forming colour-colour region, at the massive end of the overall spec-IR sample. Specifically, the SFG population comprises the most active SFGs. The high$f_{\text {AGN }}$ sub-sample are hosted by the most massive galaxies, have the least SF activity, and occupy the high end of AGN luminosity. The host galaxies of the low- $f_{\text {AGN }}$ sub-sample have stellar mass and SFRs in between these two sub-samples. We do not find significant differences in radio luminosity of these sub-samples, which could be due to the radio emission from the combined AGN and SF activity. Combining these results in the two AGN sub-samples, high- $f_{\text {AGN }}$ galaxies, on average, have higher stellar mass, lower SFR, and higher $L_{\mathrm{AGN}}$. If an evolution sequence can be made following an increase of stellar mass, low- $f_{\text {AGN }}$ would evolve to high $-f_{\mathrm{AGN}}$, with their AGN becoming a larger contribution to the internal energy injection to the host galaxy and their SFR subsequently being quenched, which is consistent with the AGNdriven quenching scenario (i.e. Fabian 2012; Page et al. 2012; Lemaux et al. 2014). However, AGN on their ramp down phase, when their AGN contributions and luminosity decrease, would also fall in our low- $f_{\text {AGN }}$ sub-sample, Therefore, we should have two galaxy populations in the low- $f_{\mathrm{AGN}}$ sub-sample, one having high SFR and one with their SFR quenched due to the AGN feedback. To further test this co-evolution scenario, we mainly focus on these two sub-samples in the next half of this paper, depending on AGN and SF activity in their own sub-samples.

In the comparison of environments, SFGs tend to preferentially reside in locally dense environments, and the low- $f_{\text {AGN }}$ sub-sample shows a marginal preference for locally intermediate density region, comparing to their control samples. However, the high- $f_{\mathrm{AGN}}$ subsample does not show any difference in local environment as compared to its control samples. All sub-samples share the same global preference as their control samples that reside in the intermediate/infall regions of clusters/groups and avoid the core region. If there is an evolutionary trend between high- and low- $f_{\mathrm{AGN}}$, their similar environmental preference suggests that their environments, on average, do not change on bulk during this evolution. We will continue the analyses of environment in Section 5.2 and discussion in Section 6.2.

\section{THE CORRELATION OF SFR AND AGN LUMINOSITY}

Many studies have demonstrated that AGN activity (e.g. AGN bolometric luminosity) correlates with SF activity especially for luminous AGNs (e.g. Netzer 2009; Gürkan et al. 2015). As we show in Fig. 5, it seems like AGN luminosity is anti-correlated with SFR for the two AGN sub-samples. However, as we mentioned at the end of the last section, each sub-sample may have a mix of AGN in different phases, which might lead to an overall anti-correlation relation. Here, we attempt to investigate the correlation of AGN and SF in their own sub-samples. We plot $L_{\mathrm{AGN}}$ and $\log (\mathrm{sSFR})$ of high- $f_{\text {AGN }}$ (orange dots) and low- $f_{\text {AGN }}$ (green dots) sub-samples in the left-hand panel of Fig. 6. The green and orange open data points and lines show median values in each $\log (\mathrm{sSFR})$ bin for galaxies in the high-/low- $f_{\mathrm{AGN}}$ sub-sample, with $\sigma_{\mathrm{NMAD}} / \sqrt{n-1}$ as their errors (see Section 4.1 for the error definition). The number of bins is chosen to have a reasonable number of galaxies in each bin. In addition, we choose fixed-width bins (1.5 dex) over the full range of $\log (\mathrm{sSFR})$ as the galaxies are not uniformly distributed in sSFR due to small numbers at the low end because of the detection limit and at the high end because of the short AGN time-scales. We will further discuss this detection limit in Section 5.1. Note that SFGs are not shown in here. Some of galaxies in the SFG population show low AGN activity. However, due to the difficulty of constraining low AGN contributions, with an overestimation up to a factor of 2 for $f_{\mathrm{AGN}}<0.1$ (Ciesla et al. 2015, see also fig. 3 in Appendix B), we do not include them in these analyses.

We find that $L_{\mathrm{AGN}}$ increases with increasing $\log (\mathrm{sSFR})$ for both the low- $f_{\mathrm{AGN}}$ and high- $f_{\mathrm{AGN}}$ samples. To further formalize this result, we calculated the Spearman rank correlation coefficient, $\rho$, as well the significance of the rejection of the null hypothesis of no correlation, $p_{\rho}$. We adopt $p_{\rho}<0.05$ as the suggestive threshold and $p_{\rho}<0.005$ as $\geq 3 \sigma$ significant threshold. We find $\rho=0.51$ and 0.64 between $L_{\mathrm{AGN}}$ and $\log (\mathrm{sSFR})$ using individual galaxies in the high- and low- $f_{\mathrm{AGN}}$ sub-samples, respectively. The small $p_{\rho}$ values $\left(p_{\rho}=0.001\right.$ and 0.006 for the high- and low- $f_{\mathrm{AGN}}$ subsamples, respectively) show the rejection of the null hypothesis of a lack of correlation between the two variables at the $\gg 3 \sigma$ level. We note that the Spearman test uses the individual data points in each sub-sample, so the result does not depend on the binning.

The number of galaxies and the median values of properties in each bin are summarized in Table 5. We find that the median redshift values are different between these bins, but that the redshift is not monotonically increasing from the lower to higher sSFR bins in either sub-class (low/high- $f_{\mathrm{AGN}}$ ). The largest variation in the median redshift is $\Delta z=0.4$, which is the difference between the high- $f_{\text {AGN }}$ median-sSFR and high-sSFR sub-sample. To evaluate the possible contribution of redshift evolution in the observed difference 

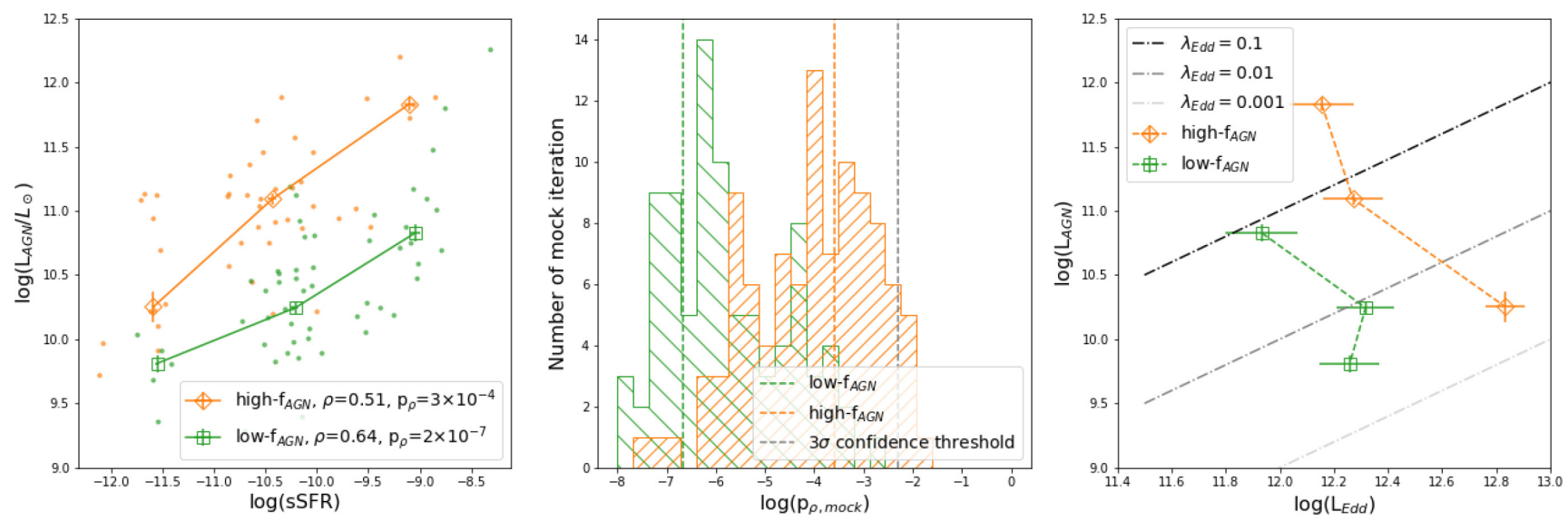

Figure 6. Left-hand panel: relation between $L_{\mathrm{AGN}}$ and $\log (\mathrm{sSFR})$ of high- $f_{\mathrm{AGN}}$ (orange) and low- $f_{\mathrm{AGN}}$ (green) sub-samples. The orange and green open data points and lines show median values in each $\log (\mathrm{sSFR})$ bin for galaxies in the high-/low- $f_{\text {AGN }}$ sub-sample, respectively. Strong and significant positive correlations between $L_{\mathrm{AGN}}$ and $\log (\mathrm{sSFR})$ are seen in both samples. Note that SFGs are not shown in here since they show no or very minimal AGN activity. Middle panel: distribution of the logarithmic value of $p_{\rho}$ between $L_{\mathrm{AGN}}$ and $\log (\mathrm{sSFR})$. This distribution is calculated from $100 \mathrm{MC}$ realizations in which we randomly assign mockf $\mathrm{AGN}_{\mathrm{An}}$ and corresponding mock- $L_{\mathrm{AGN}}$ and $-\log (\mathrm{sSFR})$ values drawn from the mock catalogues. Orange and green vertical dashed lines are $\log \left(p_{\rho}\right)$ of real sub-samples, as shown in the left-hand panel. For reference, $\operatorname{a} \log \left(p_{\rho}\right)<-2.3$ corresponds to a correlation between the two parameters estimated at $\geq 3 \sigma$ confidence level, indicated by the grey vertical dashed line. Right-hand panel: median of $L_{\mathrm{AGN}}$ and $L_{\mathrm{Edd}}$ for high- $f_{\mathrm{AGN}}$ (orange) and low- $f_{\mathrm{AGN}}$ (green) in each $\log (\mathrm{sSFR})$ bin shown in the left-hand panel. Three grey dashed lines indicate $\lambda_{\text {Edd }}=0.1,0.01$, and 0.001 from the top to bottom, respectively.

Table 5. Median properties of galaxies in high- and low- $f_{\mathrm{AGN}}$ in sSFR bins.

\begin{tabular}{|c|c|c|c|c|c|c|c|}
\hline Bin & sSFR range & Num. & $\log \left(L_{\mathrm{AGN}}\right)$ & $\log (\mathrm{SFR})$ & $\log \left(M_{*}\right)$ & $z$ & $\log \left(L_{1.4 \mathrm{GHz}}\right)$ \\
\hline High- $f_{\text {AGN }}$ median-sSFR & $-11<\mathrm{sSFR} \leq-9.5$ & 29 & $11.10 \pm 0.04$ & $0.36 \pm 0.05$ & $10.75 \pm 0.04$ & $0.87 \pm 0.03$ & $23.33 \pm 0.04$ \\
\hline Low- $f_{\text {AGN }}$ low-sSFR & $\mathrm{sSFR} \leq-11$ & 5 & $9.81 \pm 0.07$ & $-0.81 \pm 0.08$ & $10.74 \pm 0.10$ & $0.93 \pm 0.03$ & $23.52 \pm 0.10$ \\
\hline Low- $f_{\text {AGN }}$ median-sSFR & $-11<\mathrm{sSFR} \leq-9.5$ & 33 & $10.24 \pm 0.05$ & $0.63 \pm 0.04$ & $10.79 \pm 0.04$ & $0.90 \pm 0.02$ & $23.37 \pm 0.03$ \\
\hline Low- $f_{\text {AGN }}$ high-sSFR & $\mathrm{sSFR}>-9.5$ & 16 & $10.83 \pm 0.07$ & $1.35 \pm 0.08$ & $10.46 \pm 0.05$ & $1.06 \pm 0.03$ & $23.54 \pm 0.04$ \\
\hline
\end{tabular}

in the average SFR between galaxies in these bins, we use the evolution in the observed SFR- $M *$ relation for SFGs from Tomczak et al. (2016). From $z=0.87$ to 1.27 , the median redshift difference between the high- $f_{\mathrm{AGN}}$ median- and high-sSFR sub-samples, the median sSFR increases by 0.26 dex. Even the maximum possible redshift-driven evolutionary effect cannot come close to accounting for the observed difference in sSFRs between the various bins (in this case, $\sim 1.5$ dex).

To incorporate the uncertainty of $f_{\mathrm{AGN}}$ and the corresponding uncertainties in $L_{\mathrm{AGN}}$ and $\log$ (sSFR) values derived from CIGALE, we adopt the mock catalogues from the CIGALE SED fitting by varying the photometric properties (see Appendix B2). We obtain 100 sets of mock- $f_{\mathrm{AGN}}$, mock- $L_{\mathrm{AGN}}$, and mock-sSFR values for each galaxy. In each mock iteration, galaxies are then split into lowand high- $f_{\mathrm{AGN}}$ sub-samples given their mock- $f_{\mathrm{AGN}}$. A Spearman test is run on the associated mock-sSFR and mock- $\mathrm{L}_{\mathrm{AGN}}$ values to obtain a mock $p_{\rho}$. In the middle panel of Fig. 6, the histograms of mock $p_{\rho}$ of low- and high- $f_{\mathrm{AGN}}$ are shown. The correlation remains at $\gg 3 \sigma$ significant level in almost all for low- and high$f_{\text {AGN }}$ mock realizations. As confirmed by this test, it appears the positive correlation of the $L_{\mathrm{AGN}}$ and $\log (\mathrm{sSFR})$ relation observed in the high- and low- $f_{\mathrm{AGN}}$ sub-samples is a real feature of galaxies in the radio-IR that have an AGN component. If we combine the highand low- $f_{\mathrm{AGN}}$ sub-samples, the $p_{\rho}$ is 0.06 , indicating no correlation would be found. These positive significant correlations strongly suggest a co-evolution of AGN and SF in AGN found in the radioIR galaxies.

\subsection{The phases of the AGN}

Studies from observations and simulations have found that AGN duty cycles and lifetimes depend on Eddington ratio $\left(\lambda_{\text {Edd }}\right)$ and black hole mass $\left(M_{\mathrm{BH}}\right)$. Specifically, lifetimes increase as decreasing Eddington ratio and decreasing black hole mass (e.g. Martini 2004; Hopkins \& Hernquist 2009). The general picture broadly shows that the lifetime at high Eddington ratio $\left(\lambda_{\text {Edd }}>0.1\right)$ is $\sim 100 \mathrm{Myr}$ and increases rapidly at lower Eddington ratio to $\sim 0.5-1$ Gyr for $\lambda_{\text {Edd }}$ $>0.01$ and $\sim 1-5$ Gyr for $\lambda_{\text {Edd }}>0.001$. In other words, $\lambda_{\text {Edd }}$ can be used as a tracer of the AGN state and lifetime. We assume, again, that for Type $2 \mathrm{AGN}, L_{\mathrm{AGN}}$ mostly contributes to the total IR range. For a central object with $M_{\mathrm{BH}}$, the Eddington luminosity is given by Shapiro \& Teukolsky (1983):

$L_{\mathrm{Edd}}=3 \times 10^{4}\left(M_{\mathrm{BH}} / \mathrm{M}_{\odot}\right) L_{\odot}$.

We adopt the correlation of $M_{\mathrm{BH}}$ with bulge mass $M_{\text {bulge }}$ following equation (10) in Kormendy \& Ho (2013) to estimate $M_{\mathrm{BH}}$ and assume a bulge-to-total stellar mass ratio of 0.3 for galaxies $\log \left(M_{*}\right)$ $<11$ and 0.4 for $\log \left(M_{*}\right) \geq 11$ found in Lang et al. (2014), which were derived from a SFG population selected in $U V J$ colourcolour diagram at $0.5<z<2.5$. We confirm that this ratio is, on average (mean), equal to $0.3 \pm 0.03$ using 142 galaxies in the ORELSE survey. This sample occupies the star-forming region of the $N U V r J$ colour-colour diagram and has a stellar mass range of $8.3<\log \left(M_{*}\right)<11$, with a median of $10^{9.7} \mathrm{M}_{\odot}$ (Pelliccia et al. 

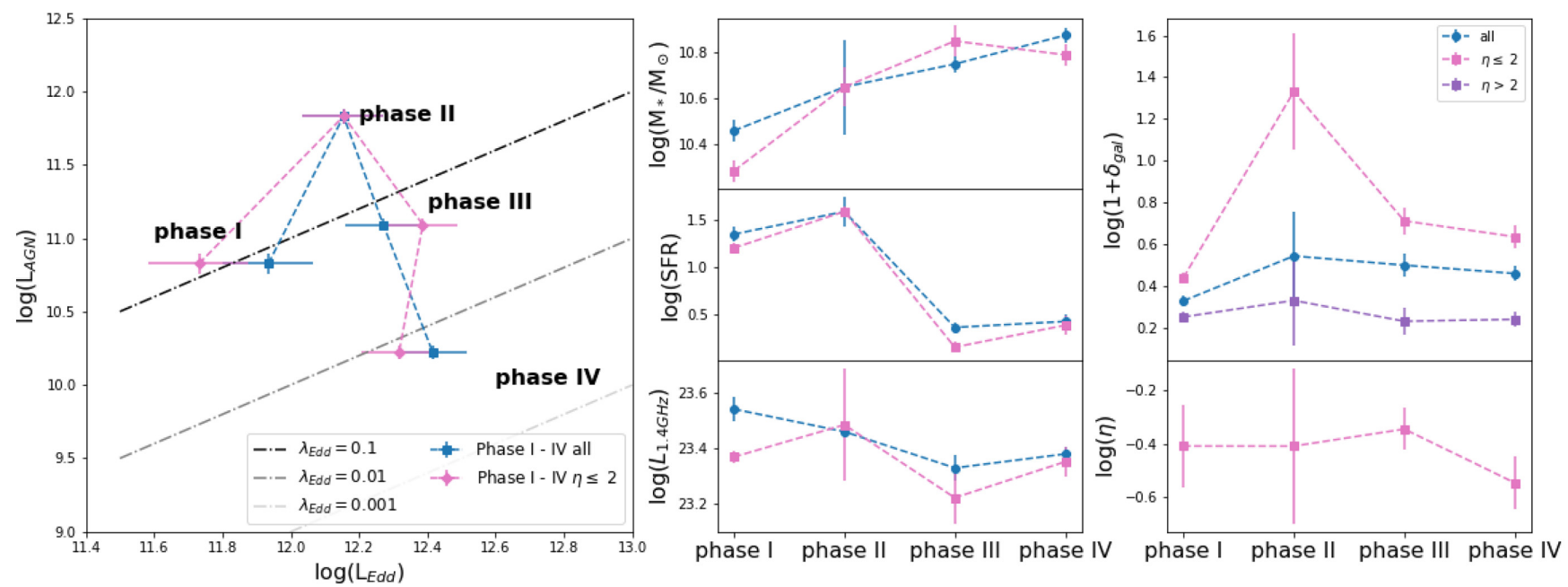

Figure 7. Left-hand panel: median $L_{\mathrm{AGN}}$ versus $L_{\mathrm{Edd}}$ of all galaxies in each phase proposed in Section 5.2 are shown in blue diamonds and those within cluster/group region $(\eta \leq 2)$ in pink squares for four phases. Middle panel (from the top to bottom): median stellar mass, SFR, radio luminosity of all galaxies in four phases and those within cluster/group regions. Top right-hand panel: median values of local overdensity of all galaxies in four phases and those within cluster/group regions and in the field. Bottom left-hand panel: median values of global environment of galaxies within cluster/group regions of the four proposed phases.

2019). ${ }^{2}$ We estimated the median $L_{\text {Edd }}$ for each bin in the lowand high- $f_{\text {AGN }}$ sub-samples binning by $\log (\mathrm{sSFR})$ as shown in the left-hand panel of Fig. 6. In the right-hand panel of Fig. 6, we plot the estimated median $\log \left(L_{\mathrm{Edd}}\right)$ versus observed $\log \left(L_{\mathrm{AGN}}\right)$ for lowand high- $f_{\mathrm{AGN}}$ bins. The uncertainty of the Eddington luminosity is estimated combining the error of stellar mass in each bin and the 0.29-dex intrinsic scatter in equation (10) in Kormendy \& Ho (2013). Note that we do not include the error from the bulge-to-total stellar mass ratio, though including the uncertainly of bulge-to-total stellar mass ratio \pm 0.1 would not affect our result.

In the right-hand panel of Fig. 6, three dashed lines from top to bottom indicate $\lambda_{\text {Edd }}=0.1,0.01$, and 0.001 , respectively, where $\lambda_{\text {Edd }}=L_{\mathrm{AGN}} / L_{\text {Edd }}$. We see that the high- $f_{\mathrm{AGN}}$ sub-sample occupies the large space from $\lambda_{\text {Edd }}=0.5$ to 0.003 , while the highest $\lambda_{\text {Edd }}$ reached in the low- $f_{\mathrm{AGN}}$ sub-sample is 0.08 . There are only 5 galaxies in the high- $f_{\mathrm{AGN}}$ high-sSFR bin at high $\lambda_{\text {Edd }}$ versus 29 in the high- $f_{\mathrm{AGN}}$ middle-sSFR bin and 16 in the low- $f_{\mathrm{AGN}}$ high-sSFR bin, with two bins at lower $\lambda_{\text {Edd }}$ region. Assuming that our sample is unbiased with respect to AGN population, the relatively small size of the high- $f_{\text {AGN }}$ high-sSFR bin might be due to the shorter lifetime at high $\lambda_{\text {Edd }}$. In addition, the number of galaxies in the low- $f_{\mathrm{AGN}}$ lowsSFR bin is small (five galaxies). One of the reason for this small sample size is likely due to the threshold of the observed $L_{\mathrm{AGN}}$. Less massive galaxies host less massive black holes and therefore lower Eddington luminosities. If they have the same AGN phase as more massive galaxies (i.e. same $\lambda_{\mathrm{Edd}}$ ), they would be detected at a lower AGN luminosity. However, due to the detection limits and the SED fitting thresholds, we are not complete for these lower AGN luminosity objects hosted by less massive galaxies. This is also seen in our data, where the stellar mass of hosts in the low$f_{\mathrm{AGN}}$ low-sSFR bin is $10^{10.74} \mathrm{M}_{\odot}$, which is lower than that in the

\footnotetext{
${ }^{2}$ The bulge-to-total stellar mass ratio is computed using the galaxy's total and spheroid component fluxes measured with the SEXTRACTOR (v2.19.5; Bertin \& Arnouts 1996; Bertin 2011), which fitted each galaxy's HST/ACS F814W image with a Sersic+exponential disc profile model convolved with the local PSF.
}

high- $f_{\text {AGN }}$ low-sSFR bin $\left(10^{11.11} \mathrm{M}_{\odot}\right)$. This might suggest that, as AGN luminosity decreases and their SF is quenched, they also fall out of the sample. We will further discuss these post-AGN and post-SFGs in Section 6.2.

\subsection{A co-evolution scenario}

To further explore the role of AGN activity in the two AGN subsamples, we propose one possible co-evolution scenario that fits our observations. We note that, due to the small sample size, a larger data set is needed to confirm this scenario. We adopt median $\lambda_{\text {Edd }}$ values as an indicator of the phases of AGN following Section 5.1 and SSFR as an indicator of SF activity. We designate galaxies in the low- $f_{\text {AGN }}$ high-sSFR bin to be 'Phase I' as they are located in the moderate $\lambda_{\text {Edd }}$ phase space and have a relatively high SFR; galaxies in high- $f_{\mathrm{AGN}}$ high-sSFR bin to be 'Phase II' as they are located in the high $\lambda_{\text {Edd }}$ phase space; galaxies in high- $f_{\mathrm{AGN}}$ middle-sSFR bin to be 'Phase III' as they are located in the moderate $\lambda_{\text {Edd }}$ phase space and have relatively low SFR; and the remaining middle-sSFR bin and low-sSFR bins to be 'Phase IV' as they are all predominantly found in the low $\lambda_{\text {Edd }}$ phase space. To determine any difference in galaxy behaviour in the cluster/group environment, we further split galaxies within the cluster/group region $(\eta \leq 2)$ as Phases I-IV $\eta$ $\leq 2$.

In the left-hand panel of Fig. 7 , the median $L_{\mathrm{AGN}}$ and $L_{\mathrm{Edd}}$ of overall galaxies in each phase are shown in blue diamonds and only those within the cluster/group region $(\eta \leq 2)$ are shown in pink squares. The median $\lambda_{\text {Edd }}$ of Phase II is the highest, which implies that AGN are in the most active state, Conversely, AGN in Phase IV are the least active. Adopting AGN lifetimes from Hopkins \& Hernquist (2009), from Phases I to III, is approximately $100 \sim$ $500 \mathrm{Myr}$ at their median estimated $M_{\mathrm{BH}} \sim 10^{7.5} \mathrm{M}_{\odot}$. The lifetime of AGN at Phase II is $<100 \mathrm{Myr}$ and the overall time-scale from Phases I to IV is $>1$ Gyr.

In the two right-hand columns of Fig. 7, we show the median stellar mass, SFR, radio luminosity, and environmental preference of all galaxies in the four phases and those within cluster/group 
Table 6. Median properties of galaxies in Phases I-IV all and within cluster/group region $(\eta \leq 2)$.

\begin{tabular}{|c|c|c|c|c|c|c|c|c|c|}
\hline Phase (all) & Num. & $\log \left(L_{\mathrm{AGN}}\right)$ & $\log \left(L_{\text {Edd }}\right)$ & $\lambda_{\text {Edd }}$ & $\log \left(M_{*}\right)$ & $\log (\mathrm{SFR})$ & $L_{1.4 \mathrm{GHz}}$ & $\log \left(1+\delta_{\text {gal }}\right)$ & $\log (\eta)$ \\
\hline Phase II & 5 & $11.83 \pm 0.05$ & $12.15 \pm 0.12$ & 0.37 & $10.65 \pm 0.21$ & $1.59 \pm 0.15$ & $23.45 \pm 0.01$ & $0.55 \pm 0.21$ & $0.005 \pm 0.66$ \\
\hline Phase IV & 50 & $10.22 \pm 0.04$ & $12.42 \pm 0.10$ & 0.007 & $10.88 \pm 0.03$ & $0.43 \pm 0.07$ & $23.38 \pm 0.03$ & $0.46 \pm 0.03$ & $1.11 \pm 0.09$ \\
\hline Phase $(\eta \leq 2)$ & Num. & $\log \left(L_{\mathrm{AGN}}\right)$ & $L_{\text {Edd }}$ & $\lambda_{\text {Edd }}$ & $\log \left(M_{*}\right)$ & $\log (\mathrm{SFR})$ & $L_{1.4 \mathrm{GHz}}$ & $\log \left(1+\delta_{\mathrm{gal}}\right)$ & $\log (\eta)$ \\
\hline Phase I & 4 & $10.83 \pm 0.07$ & $11.73 \pm 0.15$ & 0.12 & $10.29 \pm 0.05$ & $1.20 \pm 0.06$ & $23.37 \pm 0.02$ & $0.44 \pm 0.02$ & $-0.41 \pm 0.15$ \\
\hline Phase IV & 18 & $10.22 \pm 0.04$ & $12.32 \pm 0.11$ & 0.008 & $10.79 \pm 0.05$ & $0.39 \pm 0.10$ & $23.35 \pm 0.05$ & $0.64 \pm 0.05$ & $-0.55 \pm 0.10$ \\
\hline
\end{tabular}

regions. The number of galaxies in each phase and the median values of these properties are shown in Table 6 for all galaxies and galaxies in clusters/groups. We note the small number of galaxies in some Phases (e.g. Phase II overall and Phases I and II within the cluster/group region). While these phases are used to show the trends in the co-evolution scenario, a larger sample is needed to investigate these phases and further confirm this scenario.

\subsubsection{Stellar mass and SFR evolution}

In the middle-top panel of Fig. 7, we show the median stellar mass from Phases I to IV for all galaxies (blue) and galaxies in cluster/group regions (pink). A significant increase as galaxies move through these phases is shown, regardless of overall or in the cluster/group environments, which supports a co-evolution scenario.

In the centre of the middle panel, the median values of SFR are shown. It is clear that SFR drops rapidly from Phases II to III and remains constant to Phase IV. This trend suggests that from Phase I/II to later Phase III, SF must shut down within a relatively short time-scale ( $\sim 100 \mathrm{Myr}$ in Hopkins \& Hernquist 2009). Again, such a trend is seen in both the total and cluster/group galaxy sample. This result strongly suggests AGN-driven quenching in SFGs, in line with other observations in AGN hosted by SFGs selected in X-ray and IR bands (e.g. Page et al. 2012; Lemaux et al. 2014) and residual of AGN or other feedback signatures found in poststarbust galaxies (e.g. Yan et al. 2006; Kocevski et al. 2009; Lemaux et al. 2017; Rumbaugh et al. 2017). In addition, this is supported by the theory that AGN are capable of removing most of the gas from their host galaxies by winds and outflows, leaving a galaxy unable to form stars (e.g. Springel, Di Matteo \& Hernquist 2005; Hopkins, Richards \& Hernquist 2007; Somerville et al. 2008). We apply a simple test to see whether a rapid quenching is required. Given the decrease of SFR by $\sim 1$ dex, and an exponential decay $\mathrm{SFH}, \mathrm{SFR} \propto \mathrm{e}^{t / \tau}$, this would correspond to $t / \tau \approx 2.3$. For a typical short- $\tau$ model, i.e. $\tau=0.2 \mathrm{Gyr}$, it would take $\sim 500 \mathrm{Myr}$. For a longer $\tau$ model, i.e. $\tau=2 \mathrm{Gyr}$, the corresponding time-scale is 4 Gyr. However, the time-scale from Phases I to III is no more than several $500 \mathrm{Myr}$; therefore, a short- $\tau$ model is required to reach the observed decrease in SFR. In Section 6.1, we will further discuss this test, combining with proper AGN models, and quantify the quenching time-scale.

Furthermore, observations in the local universe have suggested a significant delay between the starburst phase and the peak of nuclear optical AGN activity (e.g. Davies et al. 2007; Schawinski et al. 2009; Wild, Heckman \& Charlot 2010; Yesuf et al. 2014). Indeed, in realistic hydrodynamic models, AGN luminosities do not peak at the same time as the gas density on small scales, but rather $\sim 10^{8} \mathrm{yr}$ later when most of the diffuse gas has been exhausted by SF and/or expelled by stellar feedback (Hopkins 2012).

\subsubsection{Radio luminosity evolution}

In the middle-bottom panel of Fig. 7, the median radio luminosity appears flat in AGN through this evolution scenario. There is a slight decrease from Phases I to III ( $\sim 0.1$ dex in between each phases), while such decrease is not shown to be statistically significant in the cluster/group sample, likely due to the small number of galaxies in each phase. The decrease of radio power could be due to the quenching of SF or lower radio jet power or both. In Section 4.5, we have shown that radio emission is originated from the combined AGN and SF activities. However, here we see that the SFR from Phases I to III decreases by $\sim 1 \mathrm{dex}$, corresponding to a 1-dex decrease in $L_{1.4 \mathrm{GHz}}$. The median $L_{\mathrm{AGN}}$ from Phases I to III increases by 0.2 dex, corresponding to a $\sim 0.2$-dex increase in $L_{1.4 \mathrm{GHz}}$. If we simply add these two quantities together, we are not able to obtain the $\sim 0.2$-dex difference in the radio luminosity. One reason for this finding could be due to the different time-scales associated with AGN activity and SF as well as a delay between the two (Wild et al. 2010). White et al. (2017) studied radio galaxies at $z$ $\sim 1$ down to $L_{1.4 \mathrm{GHz}} \sim 10^{22.5} \mathrm{~W} \mathrm{~Hz}^{-1}$. They found that, in the early stages of AGN-SF co-evolution, radio luminosity is dominated by emission associated with SF because the SF has not been quenched and the radio jets have not been fuelled. In the later stages, radio luminosity is taken over by AGN when the SF is being quenched and progressively lower accretion rates allow radio jets to form.

\subsubsection{Environmental evolution}

In the right-hand panel of Fig. 7, we show the environments associated with the four phases. In the top right-hand panel of Fig. 7, the median values of local overdensity of all galaxies (blue), galaxies in clusters/groups ( $\eta \leq 2$ in pink) and galaxies in the field ( $\eta>2$ in purple) in each phase. From Phases I to IV, there is a general movement of the galaxy populations to move to more locally dense environments and, in the case of phase IV, possibly to globally denser environments. These galaxies, at least those in the clusters and groups, still predominantly remain in the outskirts/infall region of their parent structure, not in the core, for the entirety of this process. Indeed, galaxies in Phase III show a higher local density than Phase I at $3 \sigma$ for all galaxies and $3.7 \sigma$ for galaxies in clusters/groups $(\log (\eta) \leq 2)$, but no significant offset is seen in the field. We see that galaxies in clusters/groups in Phase II show a higher local density, though the small sample size makes it difficult 
to tell if this attributable simply to selection effects or a statistical fluctuation.

In the bottom right-hand panel of Fig. 7, we plot the median values of global environment for galaxies in clusters/groups across the phases. All phases tend to preferentially reside in the infall region, with Phase IV being slightly closer to the core region at $2 \sigma$ significance compared to Phase III. However, due to the small number of galaxies and the associated large errors in $\log (\eta)$, we do not observe any significant change in terms of global environmental preference.

After Phase IV, galaxies might have completely quenched their SF and have lower AGN luminosity, so that they fall out of the sample completely. In order to compensate for this relative lack of galaxies in our samples, we introduce the study of Lemaux et al. (2019), which uses $~ 5000$ spectroscopically confirmed galaxies in the 15 ORELSE fields including the eight fields in this study and at the same redshift range. Galaxies with the stellar mass of the average Phase IV galaxies are right at the interface of the intermediate and high $\log \left(1+\delta_{\text {gal }}\right)$ in the high stellar mass sample where galaxies are found predominately quenched at all redshift probed by ORELSE (see fig. 5 in Lemaux et al. 2019), while such phenomenon is not seen for galaxies in other phases. This is consistent with our hypothesis that these galaxies are almost certainly on their way to becoming quiescent. These galaxies would then tend to be in the cluster/group core regions as massive quiescent galaxies. We will further discuss the effect of environment in Section 6.2.

\section{DISCUSSION}

In Section 5, we found a positive relationship between $L_{\mathrm{AGN}}$ and $\log$ (sSFR), binned by $\log (\mathrm{sSFR})$, for both high- $f_{\mathrm{AGN}}$ and low$f_{\mathrm{AGN}}$ galaxies at the $\gg 3 \sigma$ level, which strongly suggests an evolutionary trend between AGN and SF. The relation persists when the uncertainty in $f_{\mathrm{AGN}}$ estimated in CIGALE is included. We then calculated $L_{\text {Edd }}$ and sort galaxies further into Phases I-IV according to the median Eddington ratio and SFR of each bin. We found that during this proposed evolutionary scenario, AGN activities ramp up and down, as stellar mass increases constantly and SFR decreases dramatically at the AGN activity ramp down phase, which suggests an AGN-driven feedback on SF in their hosts. In Section 6.1, we further discuss the possible co-evolution of AGN and SF using a simple toy model including an AGN history (AGNH) and different SFHs. Furthermore, during this scenario, their environments of the galaxies do not change appreciably, with the galaxies remaining mostly in the relatively local dense regions that are equivalent to the intermediate/infall regions of clusters/groups. In Section 6.2, we will discuss the role of environment in this scenario.

\subsection{A simple toy model to contextualize AGN quenching}

In this section, we create a simple toy model to contextualize the time-scale of each bin in Fig. 6 and whether SF is quenched during the evolution. In addition, since we found that during the transition between Phases I and IV, galaxies in cluster/group environments and in the field share the same $M_{*}, \mathrm{SFR}, \mathrm{AGN}$, and radio luminosity trends, we use the overall radio-IR sample as the observed galaxy population. In our model, galaxies start their SF at $t_{0}$. The AGN is ignited and peaks at $t_{1}=t_{0}+\delta t$, where $\delta t=0,250$, or $500 \mathrm{Myr}$, which corresponds to what we define as scenarios SI, SII, and SIII, respectively. Once the AGN luminosity reaches its peak, it is allowed to affect SF, in terms of immediate rapid quenching, delayed rapid quenching, or no effect. These scenarios are represented by the

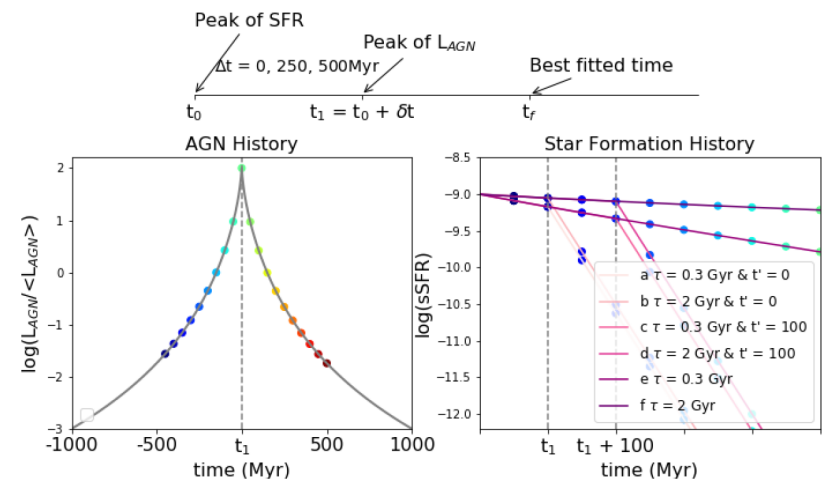

Figure 8. Top panel: a schematic illustration of toy model presented in Section 6.1. Bottom left-hand panel: the AGNH relative to the the long-term average luminosity $\left\langle L_{\mathrm{AGN}}\right\rangle$ over $2 \mathrm{Gyr}$. The luminosity distribution varies from $10^{-3}$ to $10^{2}$ relative to $\left\langle L_{\mathrm{AGN}}\right\rangle$. Bottom right-hand panel: six SFH models following an exponential decay SFH with long- $\tau=2$ and short- $\tau$ $=0.3 \mathrm{Gyr}$. Once quenching happens, we adopt a rapid- $\tau=50 \mathrm{Myr}$ model. In scenario $\mathrm{a} / \mathrm{b}$, this rapid quenching happens immediately after the peak of $L_{\mathrm{AGN}}$ at $t_{0}$; in scenario c/d, rapid quenching is delayed $t^{\prime}=100 \mathrm{Myr}$ after peak of $L_{\mathrm{AGN}}$ at $t_{0}$, while in scenario e/f, no quenching happens followed by normal short-/long- $\tau$ SFH models. The coloured points are marked at each 50-Myr time stamp.

quantity $t$ ' which is allowed to be 0,100 , or $\infty$. The illustration of this toy model is shown in the top panel of Fig. 8. We now present details of the AGNH and the SFHs adopted in this toy model.

First, we adopt the AGN luminosity distribution by integrating the Eddington ratio distributions from Hickox et al. (2014), originally from Hopkins \& Hernquist (2009), which were derived using a range of AGN observations. The AGN luminosity distribution is modelled by a Schechter function, defined as a power law with an exponential cutoff near the Eddington limit:

$\frac{\mathrm{d} t}{\mathrm{~d} \log (L)}=t_{0}\left(\frac{L}{L_{\text {peak }}}\right)^{-\alpha} \exp \left(L / L_{\text {cut }}\right)$,

where $\alpha=0.2$ and $L_{\text {peak }}=100\left\langle L_{\mathrm{AGN}}\right\rangle$ are adopted from Hickox et al. (2014), in order to include a wide diversity in AGN accretion rates and luminosity distributions that are obtained from theoretical models and observational studies of different galaxy populations. For $\alpha=0.2$ and $L_{\text {cut }}=100\left\langle L_{\mathrm{AGN}}\right\rangle$, where $\left\langle L_{\mathrm{AGN}}\right\rangle$ is the long-term average luminosity, $L_{\mathrm{AGN}}$ can reach to a lower limit $\sim 10^{-5}\left\langle L_{\mathrm{AGN}}\right\rangle$. The distribution is normalized by scaling $t_{0}$, such that the integral over $10^{-3}$ to $10^{2}$ relative to $\left\langle L_{\mathrm{AGN}}\right\rangle$ is equal to 1 . This range is chosen based on the $\sim 3$-dex $L_{\mathrm{AGN}}$ range in our sample on one end, and $\left\langle L_{\text {Edd }}\right\rangle<L_{\text {Edd }}>\sim 10^{2}\left\langle L_{\mathrm{AGN}}\right\rangle$ on the other end. We assume that the average AGNH is monotonic and symmetric. Therefore, a half-AGNH $[L(t)]$ can be obtained by inverting and integrating equation (2). We adopt a 2-Gyr AGN duty cycle, consistent with general predictions in Hopkins \& Hernquist (2009) that cover the time spent from $10^{-3}\left\langle L_{\mathrm{AGN}}\right\rangle$ through peak AGN luminosity and then back to $10^{-3}\left\langle L_{\mathrm{AGN}}\right\rangle$. This AGN luminosity distribution as a function of time is shown as the grey line in Fig. 8, bottom left-hand panel, where $t_{1}$ is the peak of AGN luminosity. We define the exact value of $\left\langle L_{\mathrm{AGN}}\right\rangle$ below.

For stellar population models, we assume exponential decay SFHs of the form SFR $\propto \mathrm{e}^{-t / \tau}$. We adopt three $\tau$ : long- $\tau=2 \mathrm{Gyr}$, short $-\tau=0.3 \mathrm{Gyr}$, and rapid- $\tau=50 \mathrm{Myr}$. It has been found that all SFGs in a given stellar mass bin and in low- to intermediate-density environments in the ORELSE survey are best fitted to $\tau=2 \mathrm{Gyr}$, for SFHs characterized to be a single $\tau$ model (Tomczak et al. 2019). We 
assume that a simulated galaxy follows a normal SFH with either a long- $\tau=2$ Gyr or a short- $\tau=0.3$ Gyr. Once quenching happens due to AGN, if it indeed occurs in the model selected, the SFH follows a rapid- $\tau=50 \mathrm{Myr}$. In scenario $\mathrm{a} / \mathrm{b}$, this rapid quenching happens immediately after the peak of $L_{\mathrm{AGN}}$ at $t_{1}$; in scenario c/d, rapid quenching is delayed $t^{\prime}=100 \mathrm{Myr}$ after peak of $L_{\mathrm{AGN}}$ at $\mathrm{t}_{0}$, motivated by a lag time between the peak of AGN luminosity and SFR (see references in Section 5.2.1), while in scenario e/f, no quenching happens and the original SFH is allowed to progress unimpeded. The amount of stellar mass generated through in situ SF in each time step is added to the previous stellar mass of the galaxy by integrating the SFR from $t=0$ to that step and subtracting the amount of stellar mass lost over that same time period resulting from stellar evolution (Moster, Naab \& White 2013). In the bottom right-hand panel of Fig. 8, the sSFR for the six SFHs models are shown with initial $\log (\mathrm{SFR})=1.5$ and $\log \left(M_{*} / \mathrm{M}_{\odot}\right)=10.5$ (see below for these choices). The coloured points are marked at each 50-Myr time stamp.

In each realization, a simulated galaxy is assigned an initial SFR and $M_{*}$ as well as a $\left\langle L_{\mathrm{AGN}}\right\rangle$, and is then allowed to evolve following all combinations of AGNH and SFH models. Since we do not know the initial SFR and stellar mass of galaxy before the AGN ignited, we use the distribution of SFR and $M_{*}$ of SFGs in the high-sSFR bin $[\log (\mathrm{sFFR})>9.5]$. Specifically, the initial state of this simulated galaxy is randomly sampled from a Gaussian distribution with the mean and variances of SFR and $M_{*}$ of this $\operatorname{bin}\left(\langle\log (\mathrm{SFR})\rangle=1.46 \pm 0.33\right.$ and $\left.\left\langle\log \left(M_{*}\right)\right\rangle=10.48 \pm 0.31\right)$. To represent the possible $L_{\mathrm{AGN}}$ of the observed galaxy population, we use the combined distribution of the estimated $L_{\mathrm{AGN}}$ of the two observed AGN sub-samples (low- and high- $f_{\text {AGN }}$ ). Specifically, we randomly assign a $\left\langle L_{\mathrm{AGN}}\right\rangle$ to this simulated galaxy sampled from a Gaussian distribution with the mean and variance of $L_{\mathrm{AGN}}\left(\left\langle L_{\mathrm{AGN}}\right\rangle=10.68 \pm 0.64\right)$. The median and $16 \mathrm{th} / 84 \mathrm{th}$ percentiles of initial sSFR and the assigned $\left\langle L_{\mathrm{AGN}}\right\rangle$ are shown as a grey triangle with error bar in Fig. 9. Note that the assigned $\left\langle L_{\mathrm{AGN}}\right\rangle$ might not be the initial state of the simulated galaxy. According to the three AGN scenarios, at $t_{0}, L_{\mathrm{AGN}}$ can be $100\left\langle L_{\mathrm{AGN}}\right\rangle, 0.22\left\langle L_{\mathrm{AGN}}\right\rangle$, or $0.02\left\langle L_{\mathrm{AGN}}\right\rangle$ in SI, SII, or SIII, respectively. Each combination of AGNH and SFH model produces a distinct set of predictions for the evolution of the sSFR and $L_{\mathrm{AGN}}$ of galaxies. We then find the best-fitting model and time-scale to observations by minimizing a residual $\chi^{2}$, defined as

$$
\begin{aligned}
& \chi^{2}=\left(\frac{\Delta \log (\mathrm{sSFR})}{\sigma \log (\mathrm{sSFR})}\right)^{2}+\left(\frac{\Delta \log \left(L_{\mathrm{AGN}}\right)}{\sigma \log \left(L_{\mathrm{AGN}}\right)}\right)^{2}, \\
& \Delta \log (\mathrm{sSFR})=\log \left(\frac{\mathrm{sSFR} \text { obs }}{\mathrm{sSFR} \text { model }}\right), \\
& \Delta \log \left(L_{\mathrm{AGN}}\right)=\log \left(\frac{L_{\mathrm{AGN}, \text { obs }}}{L_{\mathrm{AGN}, \text { model }}}\right),
\end{aligned}
$$

where $\sigma \log (\mathrm{sSFR})$ and $\sigma \log \left(L_{\mathrm{AGN}}\right)$ represent the uncertainties on the observed $\log (\mathrm{sSFR})$ and $L_{\mathrm{AGN}}$, respectively. In addition, we fit a separation line in the $\log \left(L_{\mathrm{AGN}}\right)-\log (\mathrm{sSFR})$ phase space, where above this line is the high- $f_{\mathrm{AGN}}$ model and below is the low$f_{\mathrm{AGN}}$ model. During the process of finding the best scenario for the observed points, we only consider models that lie in the same category.

In order to explore the full breadth of possible outcomes for this comparison, we run these simulations for 1000 realizations. The median of the best-fitting $\log (\mathrm{sSFR})$ and $\log \left(L_{\mathrm{AGN}}\right)$ are shown in Fig. 9 for both the high- and low- $f_{\text {AGN }}$ galaxies, plotted in comparison to the observed median $\log (\mathrm{sSFR})$ and $\log \left(L_{\mathrm{AGN}}\right)$.

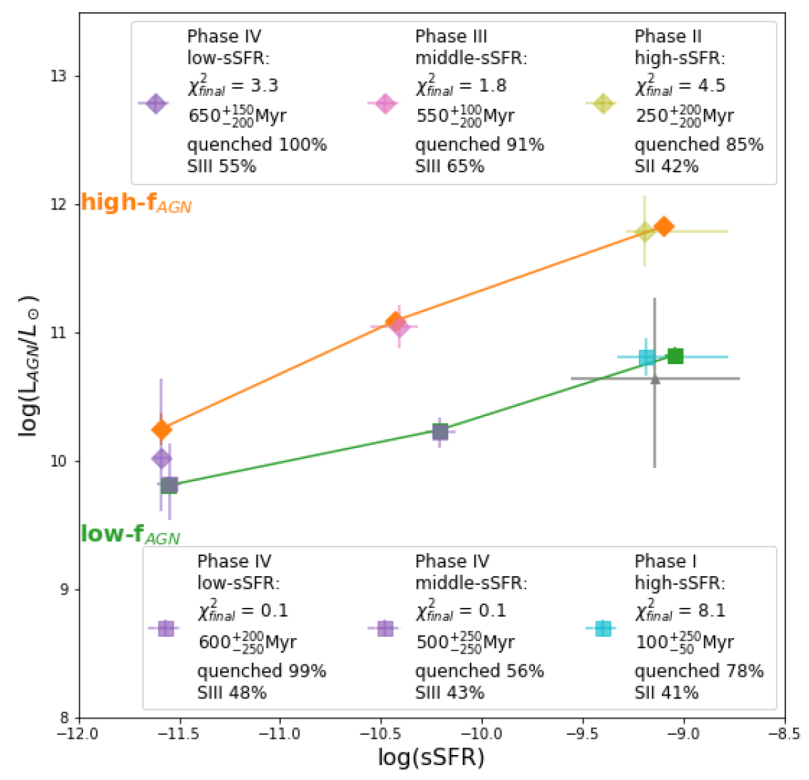

Figure 9. The median of the best-fitting $\log (\mathrm{sSFR})$ and $\log \left(L_{\mathrm{AGN}}\right)$ and the observed median $\log (\mathrm{sSFR})$ and $\log \left(L_{\mathrm{AGN}}\right)$; the same as shown in the left-hand panel of Fig. 6 . The uncertainties of the best-fitting $\log (\mathrm{sSFR})$ and $\log \left(L_{\mathrm{AGN}}\right)$ are the 16th/84th percentiles. The diamond symbols are observed and simulated data points for high $-f_{\mathrm{AGN}}$ and the squares are low- $f_{\mathrm{AGN}}$, colour coded according their phases proposed in Section 5.2. The median of initial sSFR and the assigned $\left\langle L_{\mathrm{AGN}}\right\rangle$ are shown as a grey triangle with error bar on the 16 th/ 84 th percentiles. The $\chi_{\text {final }}^{2}$ reported in the legend is calculated from the median of the best fit to the median and error of the observed data points. The median of best-fitting time with uncertainty and the preferred SFH and AGNH scenario with their percentage of simulated galaxies are listed in the legend.

The uncertainties of the best fits are the 16th/84th percentiles. The colours indicate their phases proposed in Section 5.2 and symbols according their sub-samples. The median of best-fitting $t_{\mathrm{f}}$ and 16th/84th percentiles are listed in the legend after their labels. We define the preferred scenario by counting best-fitting simulated galaxies in each AGNH and SFH scenario, separately. The most frequent one is the preferred AGNH/SFH, as shown in the legend, along with their percentage in the best-fitting simulated galaxies. In addition, we calculate a $\chi_{\text {final }}^{2}$ from the median of the best-fitting simulated models to the median and error of the observed data points in each bin.

It appears that the simulated points for the high-sSFR bins are offset to the left, with their $\chi_{\text {final }}^{2} \mathrm{~s}$ larger than other bins. This is mostly driven by the time for $L_{\mathrm{AGN}}$ to match the observations, during which SFR must decline due to the adopted exponential decay SFH. This might suggest that our toy model is not as appropriate here as it is for bins that have evolved after a longer time, mostly due to lack of information on the initial states of these phases. At longer times, such bias is migrated by simulating a large variety of initial states.

The best-fitting values of $t_{\mathrm{f}}$ are, in general, consistent with the coevolution scenario in which the best-fitting values of $t_{\mathrm{f}}$, on average, increase from Phases I to IV. However, we do see that the bestfitting $t_{\mathrm{f}}$ of Phase III and the low- $f_{\mathrm{AGN}}$ middle-sSFR bin which is in Phase IV overlap within their uncertainties. In the right-hand panel of Fig. 6 , the median $\lambda_{\text {Edd }}$ of low- $f_{\text {AGN }}$ middle-sSFR bin is shown to be the highest among the three bins included in Phase IV, indicating that they are closer to the peak of AGN and closer to Phase III. 
Furthermore, we find that in all cases, a rapid quenching $\tau$ model is preferred. For the two high-sSFR bins, though quenching scenarios are picked up more often, we find that the median $\chi^{2}$ of no quench models $\left(\tilde{\chi}^{2} \sim 7\right.$ for high- $f_{\text {AGN }}$ and $\tilde{\chi}^{2} \sim 3$ for low$\left.f_{\mathrm{AGN}}\right)$ is much lower than that of quenched models $\left(\tilde{\chi^{2}} \sim 48\right.$ for high- $f_{\mathrm{AGN}}$ and $\tilde{\chi^{2}} \sim 32$ for low $\left.-f_{\mathrm{AGN}}\right)$. Combined with the relatively short time evolved in the simulation, we are not able to draw a definite conclusion on quenching/no quenching of these bins. For the high- $f_{\text {AGN }}$ middle-sSFR bins, quenching models are picked up $\sim 90$ percent of the time, suggesting rapid quenching is required. As for the low- $f_{\text {AGN }}$ middle-sSFR bin, quenching and no quenching models are picked close to half and half inpercentage, indicating that both scenarios are equally preferred. These results suggest that the simulated galaxies can follow all AGNH/SFHs combinations and are best fitted in a wide $t_{\mathrm{f}}$ range to fit the observed point of this bin. In fact, complex types of galaxies might fall in this bin with their moderate SFR $\left(\sim 40 \mathrm{M}_{\odot} \mathrm{yr}^{-1}\right)$ and low $L_{\mathrm{AGN}}$. One possibility is a galaxy with its AGN in its ramp up phase and its SFR slowly decreasing because of gas exhaustion. It is also possible that galaxies with low luminosity AGN are not powerful enough to quench SF in their hosts. For the high-sSFR bins, in almost all cases, quenching models are picked, which strongly suggests that quenching must happen and is due to the AGN. We note that here we do not separate immediate rapid quenching $(\mathrm{a} / \mathrm{b})$ or delay rapid quenching (c/d) scenarios because the 100-Myr delay time is short; thus, the difference between having a delay or not is buried in the time of the best-fitting $t_{\mathrm{f}}$ and its uncertainties.

As for the quenching time-scale, in Phase III, simulated galaxies are dominated by scenario SIII with AGN peak at $t_{1}=500 \mathrm{Myr}$ and the quenching time-scale of $50 \mathrm{Myr}$. For low-sSFR bins of Phase IV, a longer quenching time-scale is suggested to be 100 200 Myr. We also found that all galaxies in Phase III are in the SF colour-colour region and have low $\mathrm{SFR}=2.3 \mathrm{M}_{\odot} \mathrm{yr}^{-1}$ on average, suggesting that these galaxies are on their way to quiescence. On the contrary, 65 per cent galaxies in the combined low-sSFR bins of Phase IV are in the quiescent colour-colour region with their $\log (\mathrm{sSFR}) \leq$ -11 , a signature of quiescent galaxies. Such a quenching timescale broadly agrees with studies of the observed fraction of FIRdetected host galaxies in X-ray up to $z \sim 3$ for massive galaxies $\left(\leq 10^{10} \mathrm{M}_{\odot}\right)(\sim 100 \mathrm{Myr}$; Mullaney et al. 2012; Page et al. 2012; Rosario et al. 2012). In addition, Hickox et al. (2012) found that powerful starbursts preferentially reside in the same environment as AGN at $z \sim 2$ with their estimated lifetime of $\sim 100$ Myr. They argued that powerful starbursts and AGN occur in the same systems, and AGN could be responsible for the short lifetime. In simulations, Mancuso et al. (2017) estimated $L_{1.4 \mathrm{GHz}}$ by applying the global SFR functions at different redshift and evolutionary tracks for the history of SF and $\mathrm{BH}$ accretion in an individual galaxy based on the continuity equation and the abundance matching technique. They recovered well the observed radio luminosity function in the redshift range of $0.5<z<4.5$ with $L_{1.4 \mathrm{GHz}}>10^{23.5}$ at $z \sim 1$. Their model is highly consistent with our toy model but adopted a wider rapid quenching SF $\tau$ model $(\tau \leq 10 \mathrm{Myr}$ ). We do not exclude the possibility that SF has depleted gas before the action of AGN, which is also known as a 'maintenance mode' type of feedback. In this case, it is not actually the AGN doing the quenching, but rather keeping the galaxy quenched (e.g. Croton et al. 2006). Recently, Falkendal et al. (2019) studied 25 massive RAGN with $L_{1.4 \mathrm{GHz}} \sim$ $25 \mathrm{~W} \mathrm{~Hz}^{-1}$ at $1<z<5.2$ using ALMA observation. They found that the SF in many galaxies of their sample had been quenched while AGN activity is still ongoing, which indicates that efficient SF has rapidly consumed the gas before the action of the radio jets that later removed the residual gas.

\subsection{The effect of environments}

As we found in Section 4.4, the AGN sub-samples do not show significant differences in their environmental preference either locally or globally, when comparing to the overall spec-IR sample and their control samples. They tend to be located in the intermediate/infall regions of cluster/group environments. This preference persists during the proposed co-evolution scenario (see Section 5.2). In our toy model, we found that the time-scale of this co-evolution is up to $\sim 700 \mathrm{Myr}$, which might hint that these galaxies are not infalling radially into a cluster/group, but rather have tangential orbits in their clusters/groups.

Simulations of galaxies in clusters/groups have shown that galaxies are not directly falling into the core region of the cluster/group once they are captured by the cluster/group. Indeed, galaxies may orbit beyond their host cluster virial radius, with their typical orbit time-scale of several Gyr before infalling (Wetzel et al. 2013). A short orbiting time-scale $(\sim 0.5 \mathrm{Gyr})$ is suggested at higher redshift $z$ $\sim 1$ (Muzzin et al. 2014). The tangential orbital motion of galaxies is also supported by the 'delay-and-rapid' quenching scenario recently studied in the ORELSE survey. Specifically, environmental-driven quenching efficiencies and associated time-scales are constrained in the ORELSE survey using $~ 5000$ spectroscopically confirmed galaxies in the redshift range $0.55 \leq z \leq 1.4$ (Lemaux et al. 2019). The average time-scale for SFGs in the field to be accreted by a cluster/group and become quiescent is $2.4 \pm 0.3 \mathrm{Gyr}$ for galaxies with $\log \left(M_{*} / \mathrm{M}_{\odot}\right)>10^{10.45}$ and $3.3 \pm 0.3 \mathrm{Gyr}$ for lower stellar mass galaxies. Meanwhile, the average time-scale of SFGs to be quenched is $t_{\text {quench }}=1.1 \pm 0.3 \mathrm{Gyr}$ using the same data set (Tomczak et al. 2019). The difference between these two time-scales is the $t_{\text {delay }}$, the time between accretion and the inception of the quenching process. Such a long delay time is in excess of the average time required for galaxies to move from the outskirt of a cluster to its core region (Wetzel et al. 2013; Muzzin et al. 2014). Therefore, orbits other than purely radial are required, such as a 'splashback' population characterized by galaxies on their second passage of the cluster (e.g. Chang et al. 2018).

However, the average $t_{\text {quench }}=1.1 \pm 0.3 \mathrm{Gyr}$ seems difficult to link to the short quenching time-scale found in Section 6.1. One explanation could be due to the fact that Tomczak et al. (2019) defined quiescent galaxies by the colour-colour diagram, while in this study AGN sub-samples are hosted by galaxies in the star-forming region in the colour-colour diagram. Even at the last phase, 34 percent of AGN hosts are in the star-forming region but we argue in Section 5.2.3 that they would be on their way toward quiescence. Another possibility could be due to the difference in stellar mass. AGN hosts are dominated by massive galaxies, corresponding to the intermediate and high stellar mass bins in Tomczak et al. (2019). Meanwhile, studies have shown only feedback from luminous AGN hosted by massive galaxies $\left(M>10^{11} \mathrm{M}_{\odot}\right)$ can contribute to the mass-quenching of galaxies, while other contributions are needed for lower stellar mass galaxies (e.g. Bongiorno et al. 2016). Nevertheless, Tomczak et al. (2019) probed the general SFG population, while our AGN selected in radio galaxies may undergo more vigorous starbursts, which could also lead to more rapid gas removal.

Furthermore, we see that cluster/group galaxies in phase IV tend to be closer to the cluster/group core regions, which might hint that as galaxies move towards the core, their AGN and SF activities might decline. However, our sample is not complete at low AGN luminosity and low SFR, since both MIR and radio are sensitive to galaxies that either have ongoing SF or AGN activities. In fact, the co-evolution time-scale is broadly consistent with studies of 
post-starburst/K+A populations in clusters that have recently ended a burst of SF and/or have rapidly quenched with their $\tau=0.5-$ 1 Gyr (e.g. Dressler et al. 1999; Muzzin et al. 2014; Wu et al. 2014; Lemaux et al. 2017; Socolovsky et al. 2018). Specifically, in the ORELSE survey, Lemaux et al. (2017) studied a true poststarburst population in these LSSs, finding that it consisted of both traditionally-selected $\mathrm{K}+\mathrm{A}$ galaxies and a set of $\mathrm{K}+\mathrm{A}$ galaxies with [O II] emission originating from processes other than SF, referred as 'KAIROS' galaxies. When comparing the AGN population in Phase IV in this paper with the true $\mathrm{K}+\mathrm{A}$ population from Lemaux et al. (2017), we find that both tend to be located in the intermediate region. They also found post-starburst galaxies contain spectral features indicate a rapid cessation of SF within $\sim 300 \mathrm{Myr}$ and possible residual AGN activity, consistent with the quenching time estimated in our toy model. Therefore, we argue that AGN, as they follow their co-evolutionary tracks, will evolve into true $\mathrm{K}+\mathrm{As}$. Such lines of thought will be followed further when investigating the environments of AGN and $\mathrm{K}+\mathrm{As}$ in the full ORELSE sample over the full redshift range.

\section{CONCLUSIONS}

We have studied the properties of radio-IR hosts galaxies in eight fields at $0.55 \leq z \leq 1.30$ in the ORELSE survey. We select 179 radio-IR galaxies by using VLA $1.4-\mathrm{GHz}$ observations down to a $4 \sigma$ detection flux density limit of about $40 \mu \mathrm{Jy}$, matched using a maximum likelihood technique to our spectroscopically confirmed galaxies that are also detected at $\geq 1 \sigma$ in Spitzer/MIPS imaging. We are able to constrain the AGN and SF contributions in IR luminosity separately by using the CIGALE SED fitting. We further separated radio-IR galaxies into SFGs, low-, and high- $f_{\text {AGN }}$ subsamples primary based on the fraction of AGN contribution in total IR luminosity $\left(f_{\mathrm{AGN}}\right)$ estimated in the CIGALE SED fitting, with an additional radio luminosity cut for SFGs to exclude traditionally selected RAGN. A comparison spec-IR sample was constructed using spectroscopically confirmed galaxies detected in SPIRE/MIPS 24- $\mu \mathrm{m}$ imaging at same level as the radio-IR sample.

Our main conclusions are as follows:

(i) The host galaxies of the overall radio-IR sample are situated in the dusty star-forming colour-colour region and dominate the massive end of the spec-IR sample. Specifically, the SFG population is the least massive and has the most active SFGs among the three sub-samples. Conversely, high- $f_{\mathrm{AGN}}$ are hosted by the most massive galaxies and have the least SF activity. The host galaxies of low- $f_{\text {AGN }}$ have stellar masses and SFRs in between these two populations. No significant difference between radio luminosity of these sub-samples was found, which could be due to the combined contribution of radio emission from SF and AGN activity.

(ii) As for environmental preferences, the SFG population tends to preferentially be located in locally dense regions, low- $f_{\mathrm{AGN}}$ galaxies show a marginal preference in the locally intermediate dense regions, and high- $f_{\mathrm{AGN}}$ galaxies do not show any local environmental preference, compared to their colour-stellar mass-matched control sample. In the cluster/group environments, none of the three subsamples shows different environmental preference compared to their colour-stellar mass-matched control sample, which are found to be located in the intermediate/infall regions of clusters/groups.

(iii) A positive correlation between AGN luminosity and SSFR was observed in both the low- and high- $f_{\text {AGN }}$ sub-samples, with such correlations persisting when including the uncertainty on $f_{\mathrm{AGN}}$. This result strongly suggests a co-evolution between these two activities. Based on the estimated Eddington ratio and the observed SFR, an evolution scenario was suggested following the AGN duty cycle, during which stellar mass constantly increases, whereas SF is quenched rapidly. To contextualize this co-evolution, a toy model was created. In all cases, a rapid quenching model is preferred when matching the observed AGN luminosity and sSFR, which we argue is a signature of AGN quenching.

(iv) The environmental preference for intermediate/infall regions in clusters/groups is similar in the two AGN sub-samples and persists during the evolutionary scenario, which suggests that as these AGN are quenching SF in their hosts over a $\sim 1$ Gyr timescale, they might be in an orbital motion around the clusters/groups, rather than directly infalling into the core region.

Finally, as for what physical processes may be responsible for AGN and SF activities, it is likely that these galaxies, at least those galaxies in the groups/clusters, are induced by merging, since they are hosted by massive galaxies and are preferentially found in the intermediate/infall regions where merging activities are found to be common. Therefore, we plan to follow up our radio-IR targets with an IFS/IFU to search for signs of ongoing or recent merging activity, which might allow us to prove or disprove this hypothesis. For future studies, we plan to continue the investigation of the coeval nature of AGN and SF, using AGN detected in X-ray, optical, IR, and radio bands in the full ORELSE sample, to fully characterize the cycle of AGN activity and its feedback on its host galaxy. Together with the well-constructed cluster/group catalogue in ORELSE (Hung et al. 2019), which spans a wide total dynamical mass range, we will further investigate the role of environment in the evolution of AGN, for example, as a function of cluster centric distance and parent halo mass.

\section{ACKNOWLEDGEMENTS}

This material is based upon work supported by the National Science Foundation under Grant No. 1411943. Part of the work presented herein is supported by NASA Grant Number NNX15AK92G. PFW acknowledges funding through the H2020 ERC Consolidator Grant 683184 and the support of an EACOA Fellowship from the East Asian Core Observatories Association. This study is based on data taken with the Karl G. Jansky VLA, which is operated by the National Radio Astronomy Observatory. The National Radio Astronomy Observatory is a facility of the National Science Foundation operated under cooperative agreement by Associated Universities, Inc. This work is based in part on observations made with the Spitzer Space Telescope, which is operated by the Jet Propulsion Laboratory, California Institute of Technology under a contract with NASA. SPIRE has been developed by a consortium of institutes led by Cardiff University (UK) and including Univ. Lethbridge (Canada), NAOC (China), CEA, LAM (France), IFSI, Univ. Padua (Italy), IAC (Spain), Stockholm Observatory (Sweden), Imperial College London, RAL, UCL-MSSL, UKATC, Univ. Sussex (UK), and Caltech, JPL, NHSC, Univ. Colorado (USA). This development has been supported by the following national funding agencies: CSA (Canada), NAOC (China), CEA, CNES, CNRS (France), ASI (Italy), MCINN (Spain), SNSB (Sweden), STFC, UKSA (UK), and NASA (USA). This work is based in part on data collected at the Subaru Telescope and obtained from the SMOKA, which is operated by the Astronomy Data centre, National Astronomical Observatory of Japan; observations made with the Spitzer Space Telescope, which is operated by the Jet Propulsion Laboratory, California Institute of Technology under a contract 
with NASA; and data collected at UKIRT, which is supported by NASA and operated under an agreement among the University of Hawaii, the University of Arizona, and Lockheed Martin Advanced Technology centre; operations are enabled through the cooperation of the East Asian Observatory. When the data reported here were acquired, UKIRT was operated by the Joint Astronomy Centre on behalf of the Science and Technology Facilities Council of the UK. This study is also based, in part, on observations obtained with WIRCam, a joint project of CFHT, Taiwan, Korea, Canada, France, and the Canada-France-Hawaii Telescope, which is operated by the National Research Council (NRC) of Canada, the Institut National des Sciences de l'Univers of the Centre National de la Recherche Scientifique of France, and the University of Hawai'i. The scientific results reported in this paper are based in part on observations made by the Chandra X-ray Observatory and data obtained from the Chandra Data Archive. The spectrographic data presented herein were obtained at the WM Keck Observatory, which is operated as a scientific partnership among the California Institute of Technology, the University of California, and the National Aeronautics and Space Administration. The Observatory was made possible by the generous financial support of the WM Keck Foundation. We wish to thank the indigenous Hawaiian community for allowing us to be guests on their sacred mountain, a privilege without which this work would not have been possible. We are most fortunate to be able to conduct observations from this site.

\section{REFERENCES}

Alexan der D. M., Hickox R. C., 2012, New Astron. Rev., 56, 93

Ascaso B., Lemaux B. C., Lubin L. M., Gal R. R., Kocevski D. D., Rumbaugh N., Squires G., 2014, MNRAS, 442, 589

Azadi M. et al., 2015, ApJ, 806, 187

Balogh M. L. et al., 2016, MNRAS, 456, 4364

Bardelli S. et al., 2010, A\&A, 511, A1

Bell E. F., McIntosh D. H., Katz N., Weinberg M. D., 2003, ApJS, 149, 289

Bertin E., 2011, in Evans I. N., Accomazzi A., Mink D. J., Rots A. H., eds, ASP Conf. Ser. Vol. 442, Astronomical Data Analysis Software and Systems XX. Astron. Soc. Pac., San Francisco, p. 435

Bertin E., Arnouts S., 1996, A\&AS, 117, 393

Best P. N., 2004, MNRAS, 351, 70

Best P. N., Heckman T. M., 2012, MNRAS, 421, 1569

Best P. N., Kauffmann G., Heckman T. M., Brinchmann J., Charlot S., Ivezić Ž., White S. D. M., 2005, MNRAS, 362, 25

Best P. N., Ker L. M., Simpson C., Rigby E. E., Sabater J., 2014, MNRAS, 445, 955

Bongiorno A. et al., 2016, A\&A, 588, A78

Boquien M., Buat V., Perret V., 2014, A\&A, 571, A72

Boquien M. et al., 2016, A\&A, 591, A6

Boquien M., Burgarella D., Roehlly Y., Buat V., Ciesla L., Corre D., Inoue A. K., Salas H., 2019, A\&A, 622, A103

Brightman M. et al., 2017, ApJ, 844, 10

Bruzual G., Charlot S., 2003, MNRAS, 344, 1000

Buat V. et al., 2015, A\&A, 577, A141

Calzetti D., Armus L., Bohlin R. C., Kinney A. L., Koornneef J., StorchiBergmann T., 2000, ApJ, 533, 682

Chabrier G., 2003, PASP, 115, 763

Chang C. et al., 2018, ApJ, 864, 83

Chang Y.-Y. et al., 2017, ApJS, 233, 19

Chen C.-T. J. et al., 2013, ApJ, 773, 3

Chiaberge M., Marconi A., 2011, MNRAS, 416, 917

Churazov E., Sazonov S., Sunyaev R., Forman W., Jones C., Böhringer H., 2005, MNRAS, 363, L91

Ciesla L. et al., 2015, A\&A, 576, A10

Ciotti L., Ostriker J. P., Proga D., 2010, ApJ, 717, 708
Condon J. J., 1992, ARA\&A, 30, 575

Condon J. J., Kellermann K. I., Kimball A. E., Ivezić Ž., Perley R. A., 2013, ApJ, 768, 37

Cowley M. J. et al., 2016, MNRAS, 457, 629

Croton D. J. et al., 2006, MNRAS, 365, 11

Dale D. A., Helou G., 2002, ApJ, 576, 159

Dale D. A., Helou G., Magdis G. E., Armus L., Díaz-Santos T., Shi Y., 2014, ApJ, 784, 83

Davies R. I., Müller Sánchez F., Genzel R., Tacconi L. J., Hicks E. K. S., Friedrich S., Sternberg A., 2007, ApJ, 671, 1388

Dressler A., 1980, ApJ, 236, 351

Dressler A., Smail I., Poggianti B. M., Butcher H., Couch W. J., Ellis R. S., Oemler A., Jr, 1999, ApJS, 122, 51

Ellison S. L., Patton D. R., Mendel J. T., Scudder J. M., 2011, MNRAS, 418, 2043

Fabian A. C., 2012, ARA\&A, 50, 455

Falkendal T. et al., 2019, A\&A, 621, A27

Fritz J., Franceschini A., Hatziminaoglou E., 2006, MNRAS, 366, 767

Fukugita M., Ichikawa T., Gunn J. E., Doi M., Shimasaku K., Schneider D. P., 1996, AJ, 111, 1748

Gal R. R., Lemaux B. C., Lubin L. M., Kocevski D., Squires G. K., 2008, ApJ, 684, 933

Gofford J., Reeves J. N., McLaughlin D. E., Braito V., Turner T. J., Tombesi F., Cappi M., 2015, MNRAS, 451, 4169

Grützbauch R. et al., 2011, MNRAS, 418, 938

Gürkan G. et al., 2015, MNRAS, 452, 3776

Haas M., Willner S. P., Heymann F., Ashby M. L. N., Fazio G. G., Wilkes B. J., Chini R., Siebenmorgen R., 2008, ApJ, 688, 122

Hardcastle M. J., Krause M. G. H., 2013, MNRAS, 430, 174

Harrison C. M. et al., 2012, MNRAS, 426, 1073

Heckman T. M., Best P. N., 2014, ARA\&A, 52, 589

Hickox R. C., Alexander D. M., 2018, ARA\&A, 56, 625

Hickox R. C. et al., 2009, ApJ, 696, 891

Hickox R. C. et al., 2012, MNRAS, 421, 284

Hickox R. C., Mullaney J. R., Alexander D. M., Chen C.-T. J., Civano F. M., Goulding A. D., Hainline K. N., 2014, ApJ, 782, 9

Hoaglin D. C., Mosteller F., Tukey J. W., 1983, Understanding Robust and Exploratory Data Anlysis

Hopkins P. F., 2012, MNRAS, 420, L8

Hopkins P. F., Hernquist L., 2009, ApJ, 698, 1550

Hopkins P. F., Quataert E., 2010, MNRAS, 407, 1529

Hopkins P. F., Richards G. T., Hernquist L., 2007, ApJ, 654, 731

Hopkins P. F., Torrey P., Faucher-Giguère C.-A., Quataert E., Murray N., 2016, MNRAS, 458, 816

Hopkins A. M. et al., 2003, ApJ, 599, 971

Ho L. C., 2002, ApJ, 564, 120

Hung D. et al., 2019, MNRAS, 491, 5524

Hurley P. D. et al., 2017, MNRAS, 464, 885

Ibar E., Ivison R. J., Best P. N., Coppin K., Pope A., Smail I., Dunlop J. S., 2010, MNRAS, 401, L53

Ilbert O. et al., 2006, A\&A, 457, 841

Ishibashi W., Auger M. W., Zhang D., Fabian A. C., 2014, MNRAS, 443, 1339

Kauffmann G. et al., 2003, MNRAS, 346, 1055

Kaviraj S., Kirkby L. A., Silk J., Sarzi M., 2007, MNRAS, 382, 960

Kennicutt R. C., Jr, 1998, ARA\&A, 36, 189

Kimball A. E., Kellermann K. I., Condon J. J., Ivezić Ž., Perley R. A., 2011, ApJ, 739, L29

Kocevski D. D., Lubin L. M., Lemaux B. C., Gal R. R., Fassnacht C. D., Lin R., Squires G. K., 2009, ApJ, 700, 901

Kormendy J., Ho L. C., 2013, ARA\&A, 51, 511

Lang P. et al., 2014, ApJ, 788, 11

Lanzuisi G. et al., 2017, A\&A, 602, A123

Laor A., 2000, ApJ, 543, L111

Ledlow M. J., Owen F. N., 1996, AJ, 112, 9

Lemaux B. C. et al., 2012, ApJ, 745, 106

Lemaux B. C. et al., 2014, A\&A, 572, A90

Lemaux B. C. et al., 2019, MNRAS, 490, 1231 
Lemaux B. C., Tomczak A. R., Lubin L. M., Wu P.-F., Gal R. R., Rumbaugh N., Kocevski D. D., Squires G. K., 2017, MNRAS, 472, 419

Lindsay S. N. et al., 2014, MNRAS, 440, 1527

Lofthouse E. K., Kaviraj S., Smith D. J. B., Hardcastle M. J., 2018, MNRAS, 479,807

Lubin L. M., Gal R. R., Lemaux B. C., Kocevski D. D., Squires G. K., 2009, AJ, 137, 4867

Lutz D. et al., 2010, ApJ, 712, 1287

McLure R. J., Jarvis M. J., 2004, MNRAS, 353, L45

Magliocchetti M. et al., 2004, MNRAS, 350, 1485

Magliocchetti M., Popesso P., Brusa M., Salvato M., 2018, MNRAS, 473, 2493

Mancuso C. et al., 2017, ApJ, 842, 95

Martini P., 2004, in Ho L. C., ed., Carnegie Obs. Centennial Symp., Coevolution of Black Holes and Galaxies. Cambridge Univ. Press, Cambridge, p. 169

Merlin E. et al., 2015, A\&A, 582, A15

Miller N. A., Owen F. N., 2002, AJ, 124, 2453

Moster B. P., Naab T., White S. D. M., 2013, MNRAS, 428, 3121

Mullaney J. R. et al., 2012, MNRAS, 419, 95

Muzzin A. et al., 2014, ApJ, 796, 65

Netzer H., 2009, MNRAS, 399, 1907

Newman J. A. et al., 2013, ApJS, 208, 5

Ogle P., Whysong D., Antonucci R., 2006, ApJ, 647, 161

Oke J. B., Gunn J. E., 1983, ApJ, 266, 713

Padovani P., 2016, A\&AR, 24, 13

Padovani P., Miller N., Kellermann K. I., Mainieri V., Rosati P., Tozzi P., 2011, ApJ, 740, 20

Padovani P. et al., 2017, A\&AR, 25, 2

Page M. J. et al., 2012, Nature, 485, 213

Panessa F. et al., 2015, MNRAS, 447, 1289

Panessa F., Baldi R. D., Laor A., Padovani P., Behar E., McHardy I., 2019, Nat. Astron., 3, 387

Pelliccia D. et al., 2019, MNRAS, 482, 3514

Peng Y.-j. et al., 2010, ApJ, 721, 193

Peng Y.-j., Lilly S. J., Renzini A., Carollo M., 2012, ApJ, 757, 4

Pierce J. C. S., Tadhunter C. N., Ramos Almeida C., Bessiere P. S., Rose M., 2019, MNRAS, 487, 5490

Poggianti B. M. et al., 2009, ApJ, 693, 112

Read S. C. et al., 2018, MNRAS, 480, 5625

Rees G. A. et al., 2016, MNRAS, 455, 2731

Rosario D. J. et al., 2012, A\&A, 545, A45

Roseboom I. G. et al., 2010, MNRAS, 409, 48

Rumbaugh N. et al., 2017, MNRAS, 466, 496

Rumbaugh N., Kocevski D. D., Gal R. R., Lemaux B. C., Lubin L. M., Fassnacht C. D., McGrath E. J., Squires G. K., 2012, ApJ, 746, 155

Santini P. et al., 2012, A\&A, 540, A109

Schawinski K., Virani S., Simmons B., Urry C. M., Treister E., Kaviraj S., Kushkuley B., 2009, ApJ, 692, L19

Scholtz J. et al., 2018, MNRAS, 475, 1288

Shapiro S. L., Teukolsky S. A., 1983, Black Holes, White Dwarfs, and Neutron Stars: The Physics of Compact Objects. Wiley, New York

Shen L. et al., 2017, MNRAS, 472, 998

Shen L. et al., 2019, MNRAS, 484, 2433

Sikora M., Begelman M. C., 2013, ApJ, 764, L24

Sikora M., Stawarz Ł., Lasota J.-P., 2007, ApJ, 658, 815

Smolčić V., 2009, ApJ, 699, L43

Socolovsky M., Almaini O., Hatch N. A., Wild V., Maltby D. T., Hartley W. G., Simpson C., 2018, MNRAS, 476, 1242

Somerville R. S., Hopkins P. F., Cox T. J., Robertson B. E., Hernquist L., 2008, MNRAS, 391, 481

Springel V., Di Matteo T., Hernquist L., 2005, MNRAS, 361, 776

Stanley F., Harrison C. M., Alexander D. M., Swinbank A. M., Aird J. A., Del Moro A., Hickox R. C., Mullaney J. R., 2015, MNRAS, 453, 591

Stern D. et al., 2012, ApJ, 753, 30

Tadhunter C., 2016, A\&AR, 24, 10

Tomczak A. R. et al., 2016, ApJ, 817, 118

Tomczak A. R. et al., 2017, MNRAS, 472, 3512

Tomczak A. R. et al., 2019, MNRAS, 484, 4695
Valiante E. et al., 2016, MNRAS, 462, 3146

Wang L. et al., 2014, MNRAS, 444, 2870

Wetzel A. R., Tinker J. L., Conroy C., van den Bosch F. C., 2013, MNRAS, 432,336

White S. V., Jarvis M. J., Häußler B., Maddox N., 2015, MNRAS, 448, 2665

White S. V., Jarvis M. J., Kalfountzou E., Hardcastle M. J., Verma A., Cao Orjales J. M., Stevens J., 2017, MNRAS, 468, 217

Whysong D., Antonucci R., 2004, ApJ, 602, 116

Wild V., Heckman T., Charlot S., 2010, MNRAS, 405, 933

Williams R. J., Quadri R. F., Franx M., van Dokkum P., Labbé I., 2009, ApJ, 691,1879

Wu P.-F., Gal R. R., Lemaux B. C., Kocevski D. D., Lubin L. M., Rumbaugh N., Squires G. K., 2014, ApJ, 792, 16

Wuyts S., Labbé I., Förster Schreiber N. M., Franx M., Rudnick G., Brammer G. B., van Dokkum P. G., 2008, ApJ, 682, 985

Yan R., Newman J. A., Faber S. M., Konidaris N., Koo D., Davis M., 2006, ApJ, 648, 281

Yesuf H. M., Faber S. M., Trump J. R., Koo D. C., Fang J. J., Liu F. S., Wild V., Hayward C. C., 2014, ApJ, 792, 84

Yuan F., Narayan R., 2014, ARA\&A, 52, 529

Yun M. S., Reddy N. A., Condon J. J., 2001, ApJ, 554, 803

Zakamska N. L. et al., 2016, MNRAS, 455, 4191

\section{APPENDIX A: T-PHOT SIMULATION TEST}

To check the performance of T-PHOT on Herschel/SPIRE images, we run a test on a simulated Herschel/SPIRE 250- $\mu$ m image $\left(\mathrm{FWHM}=25\right.$ and $3.6 \operatorname{arcsec}$ pixel $\left.^{-1}\right)$. We begin with a $30 \times 30 \mathrm{arcmin}^{2}$ field of view and populate it with $\sim 18,000$ simulated objects. Each object is a 2D Gaussian with a randomly assigned position, size, ellipticity, and position angle. Herschel/SPIRE 250$\mu \mathrm{m}$ fluxes are assigned to each of these objects in order to reproduce the observed flux density function from H-ATLAS in Valiante et al. (2016); because of the limited depth of HATLAS ( $25 \mathrm{mJy}$ ) and the small simulated field of view, we fit a power law to the $250-\mu$ m flux density function and extrapolate down to $2 \mathrm{mJy}$. Next, we convolve the simulated objects with the Herschel/SPIRE 250- $\mu$ m PSF. For the final step, noise is added to the images sampled from a Gaussian distribution and set to reproduce the background $\mathrm{rms} \sim 2.5 \mathrm{mJy}$ of the 250- $\mu \mathrm{m}$ images of XLSS005.

For this test, T-PHOT was run using the list of simulated object positions as unresolved priors with cells-on-objects method. The results of the relative flux difference $\left(f_{\text {measured }}-f_{\text {true }}\right) / f_{\text {true }}$ versus the

Table A1. Depth of imaging data.

\begin{tabular}{|c|c|c|c|c|c|}
\hline $\begin{array}{l}\text { Field } \\
\text { Wavelegth } \\
\text { Units }\end{array}$ & $\begin{array}{c}\operatorname{MIPS}^{a} \\
24 \mu \mathrm{m} \\
(\mu \mathrm{Jy})\end{array}$ & $\begin{array}{l}\mathrm{PSW}^{b} \\
250 \mu \mathrm{m} \\
(\mathrm{mJy})\end{array}$ & $\begin{array}{c}\text { PMW } \\
350 \mu \mathrm{m} \\
(\mathrm{mJy})\end{array}$ & $\begin{array}{c}\text { PLW } \\
500 \mu \mathrm{m} \\
(\mathrm{mJy})\end{array}$ & $\begin{array}{c}\mathrm{VLA}^{c} \\
1.4 \mathrm{GHz} \\
(\mu \mathrm{Jy})\end{array}$ \\
\hline RX J1716 & $65.4(33.6)$ & - & - & - & 15.2 \\
\hline RX J1821 & 39.7 (11.4) & - & - & - & 9.3 \\
\hline SG0023 & $4.1(0.8)$ & 12.4 & 18.9 & 22.4 & 13.9 \\
\hline SC1604 & $9.4(3.6)$ & - & - & - & 9.30 \\
\hline XLSS005 & $184.6(90.1)$ & 5.9 & 13.5 & 14.2 & 14.1 \\
\hline SC0910 & 74.4 (29.9) & 8.5 & 11.6 & 14.6 & 14.7 \\
\hline RX J1053 & $41.2(16.0)$ & 6.7 & 15.3 & 15.1 & 10.2 \\
\hline $\mathrm{C} 11350$ & & - & - & - & 9.8 \\
\hline C11429 & & - & - & - & 17.1 \\
\hline
\end{tabular}

Notes. ${ }^{a} 3 \sigma(1 \sigma)$ point source completeness limit.

${ }^{b} 1 \sigma$ detection limit of 250-, 350-, and 500- $\mu \mathrm{m}$ Herschel/SPIRE images as described in Section 2.2. Note that the reported detection limits represent the upper limits in these highly confused images.

${ }^{c}$ rms sensitivity for final image associated with all data for that pointing. Note that radio sources are selected with a significance $\geq 4$ times rms in either the radio-integrated flux or peak flux. 


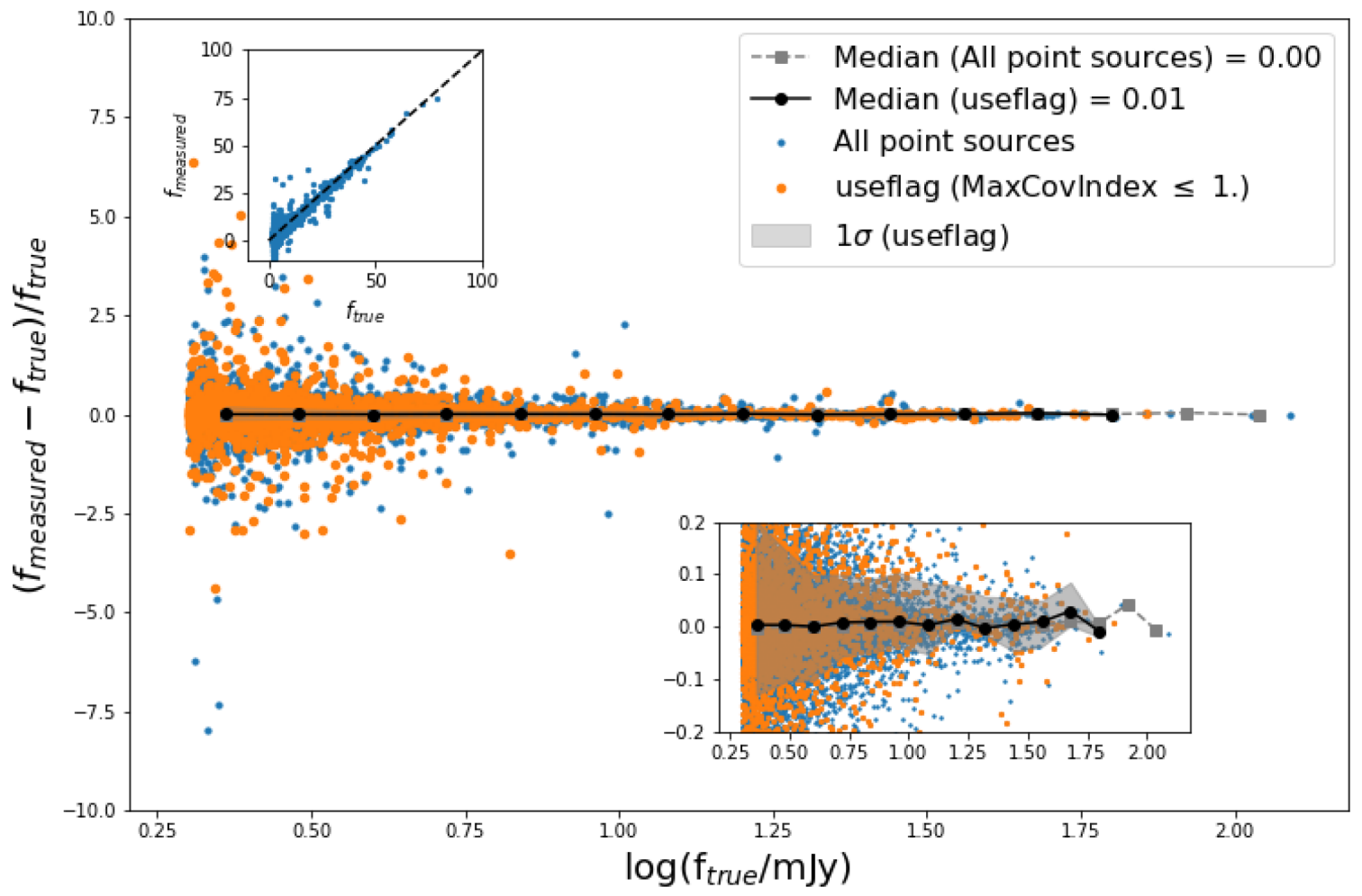

Figure A1. Accuracy of the flux determination in a FIR-like simulation (Herschel/SPIRE 250- $\mu \mathrm{m}, \mathrm{FWHM}=25$ and 3.6 arcsec pixel ${ }^{-1}$ ), using unresolved priors. The median of the relative flux difference $\left(f_{\text {measured }}-f_{\text {true }}\right) / f_{\text {true }}$ is close to zero, while the scatter of differences increases for low flux objects. We are confidence with the flux measured in this way down to $\sim 3 \mathrm{mJy}$ where the spread of the relative flux differences is $<0.1$. This threshold is lower than the $1 \sigma$ depth of our Herschel/SPIRE 250- $\mu \mathrm{m}$ image shown in A1.

$\log$ of the real input flux $f_{\text {true }}$ is plotted in Fig. A1. The colour of the symbols refer to a proxy for a covariance index 'MaxCovIndex', which is an indicator of the reliability of the fit. Large covariances often indicate a possible systematic offset in the measured flux of objects. The median of the relative flux differences is shown in the black solid dots, connected by the solid line, binning by simulated fluxes. The median is consistent with zero down to the faintest fluxes, independent of the simulated fluxes. This result shows that T-PHOT can recover the input fluxes of the sources with great statistical accuracy. The spread of the relative flux differences (16th/84th percentiles) is $\sim 0.1$ for simulated objects at $\sim 3 \mathrm{mJy}$, dropping to $\sim 0.05$ for simulated objects at $\sim 10 \mathrm{mJy}$. Therefore, we are confident that we can measure fluxes of objects with fluxes $>3 \mathrm{mJy}$. In addition, we found a negligible offset on the median of detection having large covariances index. Therefore, we do not include this threshold in this paper.

\section{APPENDIX B: UNCERTAINTY FROM CIGALE}

\section{B1 Bias from CIGALE}

Here we compare CIGALE-derived stellar mass to FAST-derived stellar mass, and CIGALE-derived SFR to the SFR estimated by adding contributions from obscured and unobscured young stellar populations as traced by IR and UV emission, respectively $\left(\mathrm{SFR}_{\mathrm{UV}+\mathrm{IR}}\right.$; Tomczak et al. 2019).

In the top panel of Fig. B1, we show the differences in stellar mass derived from CIGALE and those derived from FAST, for the overall radio-IR galaxies (grey dots) and those that are both above the stellar mass cut and have good fits in CIGALE $\left(M_{*}>10^{10} \mathrm{M}_{\odot}\right.$ and $\chi^{2} \leq 10$; black dots). The latter sample is the final sample analysed in this paper. The median of difference is $-0.04_{-0.21}^{+0.12}$ with the scatter calculated from the 16 th/ 84 th percentiles. The difference is small and remains constant across the full stellar mass range that studied in this paper. Note that we use stellar masses derived from FAST in this paper in order to perform comparison to the overall spectroscopically confirmed sample.

In the bottom panel, we show the differences in SFR derived from CIGALE and those estimated from $\mathrm{SFR}_{\mathrm{UV}+\mathrm{IR}}$. The median is $-0.63_{-1.34}^{-0.22}$ with the scatter calculated from the 16 th $/ 84$ th percentiles. A systematically higher value of $\mathrm{SFR}_{\mathrm{UV}+\mathrm{IR}}$ is seen, which could due to other sources of UV/dust heating (such as A or F stars) that are not tied to SF increases in the IR luminosity and cause an overestimation of the true SFR (see Boquien et al. 2014, 2016 for more discussions). Actually, similar trends are also seen when comparing SFR derived from FAST and LEPHARE (Ilbert et al. 2006) versus SFR estimated from the UV+IR method with the offset increasing at low SFR. This is a feature of SFR estimated from SED modelling, which allow SFH with low sSFR $\sim 0$, while $\mathrm{SFR}_{\mathrm{UV}+\mathrm{IR}}$ will always be $>0$. Therefore, the offset is larger for lower SFR.

\section{B2 Comparison with physical quantities derived from the mock catalogue}

In this section, we adopt the procedure of generating mock catalogues in CIGALE, in order to check the reliability of estimated parameters. In brief, the mock photometric data for each object is modified by adding a value taken from a Gaussian distribution using the photometric error as the standard deviation. Then a mock 


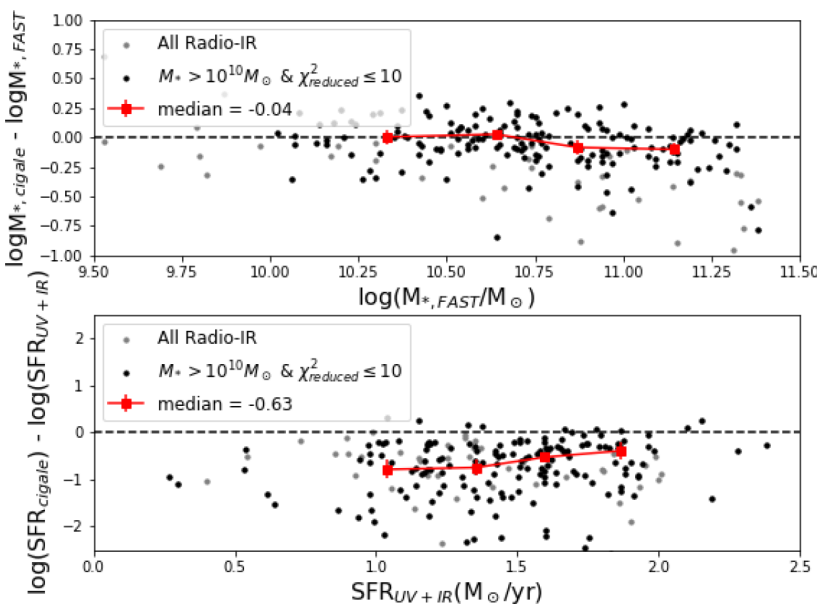

Figure B1. Comparison with physical quantities derived from CIGALE and other method for the overall radio-IR galaxies (grey dots) and those above the stellar mass cut $\left(M_{*}>10^{10} \mathrm{M}_{\odot}\right)$ and good fitted in CIGALE $\left(\chi^{2} \leq 10\right)$ shown as black dots. The latter sample is the final sample analysed in this paper. Top panel: difference of stellar mass derived from CIGALE and derived from FAST as a function of the latter quantity. Note that we use stellar mass derived from FASTT in this paper, in order to perform comparisons to the overall spectroscopically confirmed sample. The median of the difference is $-0.04_{-0.21}^{+0.12}$ with the scatter calculated from the 16 th $/ 84$ th percentiles. The difference is small and remain constant across the full stellar mass range studied in this paper. Bottom panel: difference of SFR derived from CIGALE and estimated by adding contributions from obscured and unobscured young stellar populations as traced by IR and UV emission respectively $\left(\mathrm{SFR}_{\mathrm{UV}+\mathrm{IR}}\right.$; Tomczak et al. 2019), as a function of the latter quantity. The median is $-0.63_{-1.34}^{-0.22}$ with the scatter calculated from the $16 \mathrm{th} / 84$ th percentiles. The systematic higher value of $\mathrm{SFR}_{\mathrm{UV}+\mathrm{IR}}$ is due to other sources of UV/dust heating (such as A or F stars) that are not tied to SF increases in the IR luminosity and cause an overestimation of true SFR (see Boquien et al. 2014, 2016 for more discussions).

catalogue is obtained by running CIGALE on the same parameter space and same method as the real catalogue (see Boquien et al. 2019 for more details). In addition, we generate 100 mocks for each radio-IR galaxy. Fig. B2 shows the differences ( $\Delta=$ mock - real) in $\log \left(M_{*}\right), \log (\mathrm{SFR}), L_{\mathrm{IR}}, f_{\mathrm{AGN}}, L_{\mathrm{AGN}}$ derived from mock catalogues and those derived from the real catalogue as a function of real values and their histograms in the right-hand panels. The median values of $\Delta \log \left(M_{*}\right), \Delta \log (\mathrm{SFR}), \Delta L_{\mathrm{IR}}, \Delta f_{\mathrm{AGN}}$, and $\Delta L_{\mathrm{AGN}}$ are $\sim 0$.

The largest difference is shown in the $f_{\mathrm{AGN}}$ panel. At the low$f_{\mathrm{AGN}}$ end, the $f_{\mathrm{AGN}}$ from mocks are lower than the real value. The differences appear to increase with increasing $f_{\mathrm{AGN}}$, indicating that CIGALE may underestimate the low $f_{\mathrm{AGN}}$ and overestimate high $f_{\mathrm{AGN}}$. The scatter is large with differences from -1 to +1 ; however, the median is $0 \pm 0.1$ with uncertainty on 16 th/84th percentiles. Therefore, we trust the determination of $f_{\text {AGN }}$ to a difference of 0.2 based on the 16th/84th percentiles. We note that the separation of our AGN sub-samples is larger than this reliable difference and the median $f_{\mathrm{AGN}}$ of our low- and high- $f_{\mathrm{AGN}}$ sub-samples is 0.2 and 0.8 , respectively. As such, this lack of precision to \pm 0.2 in $f_{\mathrm{AGN}}$ does not affect any result shown in this paper. In fact, we included the uncertainty of $f_{\mathrm{AGN}}$ in Section 4.7, which does not change our result.
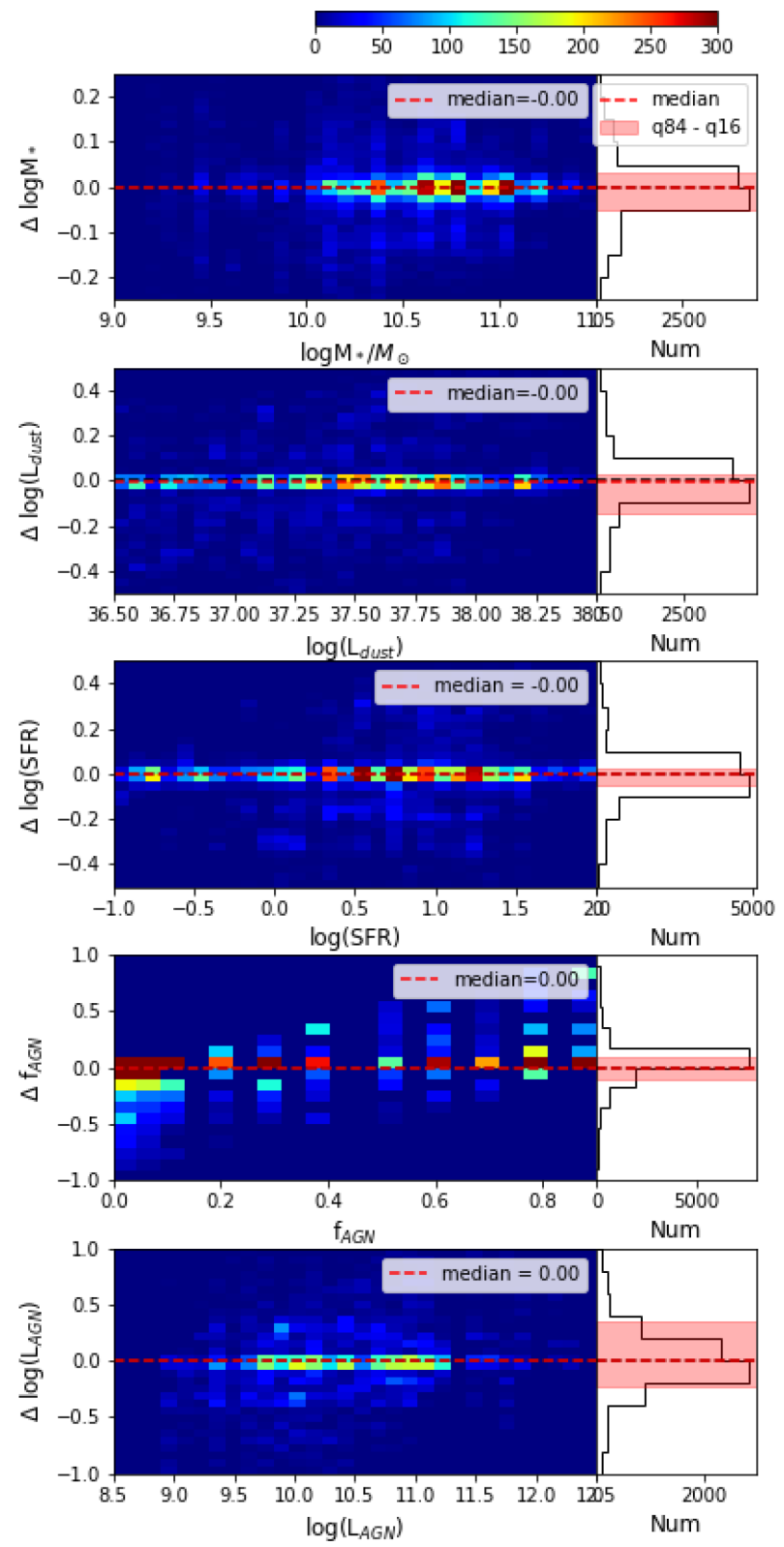

Figure B2. Comparison with physical quantities derived from 100 mocks of each radio-IR galaxy compared to the real catalogue. Left-hand panel: 2D histograms of the difference of quantities estimated in mock versus that estimated in the real catalogue, as a function of the real quantities, colour coded by the number of mocks in the $2 \mathrm{D}$ box. The same colour convention is used for all right-hand panels as shown in the top of the figure. From top to bottom panels are stellar mass, $\log \left(L_{\mathrm{dust}}\right), \mathrm{SFR}, f_{\mathrm{AGN}}$, and $L_{\mathrm{AGN}}$. The median and 16th/84th percentiles of the differences are listed in the legend. Right-hand panel: histogram of the difference in the left-hand panel. The median of the difference is marked in the red horizontal dashed line with the shaded red region corresponding to the spread of 16th and 84th percentiles. The median values of differences are 0 , suggesting small uncertainties of these quantity.

This paper has been typeset from a $\mathrm{T}_{\mathrm{E}} \mathrm{X} / \mathrm{L} \mathrm{A} \mathrm{E} \mathrm{X}$ file prepared by the author. 